\title{
To what extent is social inequality in school-age achievement determined before and during schooling? A longitudinal analysis in three European countries
}

\author{
Giampiero Passaretta ${ }^{a^{*}} \quad$ Jan Skopek $^{\mathrm{b}} \quad$ Thomas van Huizen $^{\mathrm{c}}$ \\ ${ }^{a}$ European University Institute, Department of Political and Social Sciences \\ ${ }^{\mathrm{b}}$ Trinity College Dublin, Department of Sociology \\ ${ }^{\mathrm{c}}$ Utrecht University, School of Economics \\ *Corresponding author: giampiero.passaretta@,eui.eu
}

Discussion paper

\begin{abstract}
We estimate the degree to which socioeconomic status (SES) gaps in children's language skills observed in primary schooling are already determined before children enter school in Germany, the Netherlands, and the United Kingdom. We use representative and longitudinal cohort data and apply instrumental variable estimation to deal with measurement error in test scores. Around $60-80 \%$ of SES gaps in language at the end of primary school are attributable to gaps settled before formal schooling, while at most $20-40 \%$ is attributable to SES operating during the school years. We also show that ignoring measurement error results in a major overestimation of the role of SES during schooling. Our findings suggest that the most effective strategy for reducing social inequality in school-age achievement is reducing inequality before school life starts.
\end{abstract}

Keywords: inequality of educational opportunity, achievement, primary school, longitudinal analysis, cross-national research, social inequality

Acknowledgments: The authors acknowledge funding from the European Union's Horizon 2020 research and innovation program under grant agreement No. 727069 (ISOTIS). 


\section{Introduction}

The association between parental socioeconomic status (hereafter: SES) and students' educational achievement has been documented in virtually all Western countries (Barone, 2006; Marks, Cresswell, \& Ainley, 2006). SES gaps in educational achievement explain a large part of social differences in educational transitions, such as track choice or school continuation (Jackson, 2013; Blossfeld et al. 2016). Hence, inequality in achievement is a major mechanism through which social inequality in educational attainment is produced. Also, SES inequality in achievement predict SES differences in later labor market outcomes over and above formal educational qualifications (Barone \& Werfhorst, 2011; Kerckhoff, Raudenbush, \& Glennie, 2001).

What is the nature of SES gaps in achievement and the process by which they evolve over children's educational career? One theoretically important but still understudied aspect of inequality of educational achievement is the concept of path-dependency by which inequality outcomes are dynamically related to previous inequality outcomes (DiPrete \& Eirich, 2006). Drawing upon data from child cohort studies, a growing body of longitudinal research examines the early roots and the evolution of SES gaps in academic achievement. A series of studies (mostly on Anglophone countries) have demonstrated quite consistently that social gaps in achievement emerge and grow before children enroll in school but remain by and large stable and only slightly increase over schooling (e.g., Bradbury, Corak, Waldfogel, \& Washbrook, 2015; Cheadle, 2009; Feinstein, 2003; Potter \& Roksa, 2013; Skopek \& Passaretta, 2018; von Hippel and Hamrock, 2019). This consistent finding may draw the attention of scholars and policymakers being concerned with inequality of educational opportunities towards the early years of life (see, for example, Farkas and Beron 2004). However, most of prior studies trace group-based trajectories of achievement but miss a dynamic perspective. Indeed, comparing achievement trajectories by SES groups may obscure the role of cumulative and pathdependent processes through which SES generates unequal achievement outcomes at different life stages (Baumert, Nagy, and Lehmann 2012; for a discussion of similar issues but related to occupational careers see Passaretta et al. 2018).

Our dynamic perspective treats inequality in achievement as a path-dependent process in which current states of inequality are partly inherited by previous states. Path-dependencies in educational inequality will cause a certain degree of persistency in inequality outcomes which bears important implications for theory building and policymaking. Let us illustrate the point by a thought experiment on two extreme scenarios (A and B). Imagine measuring relative 
inequalities in language skills at the end of kindergarten age, say the first day of grade 1, and at the end of grade 4. This would allow us to trace the evolution of the relative position of children in the distribution of language achievement over time. SES-based inequality in language skills remains constant between $1^{\text {st }}$ and $4^{\text {th }}$ grade in both Scenarios, A and B. In scenario A, social inequality in $4^{\text {th }}$ grade was just a perfect replica of social inequality formed up to $1^{\text {st }}$ grade. At both time points children retained the same relative position in the achievement distribution, thus resulting in a constant gap between high and low SES children over time. In this scenario, SES operated on the formation of achievement inequality in $4^{\text {th }}$ grade through inequalities settled before $1^{\text {st }}$ grade entirely but had played no additional role on achievement beyond grade 1 . In Scenario B, social inequality in language formed in up to $1^{\text {st }}$ grade was inconsequential for inequality in later language abilities. A child's relative achievement in $4^{\text {th }}$ grade was entirely independent of her relative achievement in the beginning of $1^{\text {st }}$ grade. This happens if SES operated on reading achievement between $1^{\text {st }}$ and $4^{\text {th }}$ grade directly and independently of earlier achievement, as earlier and later achievement were not genuinely associated. Both scenarios result in the same inequality outcomes (constant SESbased gaps between $1^{\text {st }}$ and $4^{\text {th }}$ grade) but feature fundamentally different generative processes. For social policy aimed at reducing SES-based gaps to be effective it would need to react differently to either of these scenarios.

To sum up, the patterns of stable or slightly increasing SES gaps in achievement over schooling found in the previous literature may be caused by the direct and cumulative influence of parental background unfolding over time - for example, via enduring support to children over the school career - or the indirect effect of preschool-age achievements on subsequent school-age achievements (Protopapas, Sideridis, Mouzaki, \& Panagiotis, 2011), or most likely a combination of both.

Our paper studies path-dependency in achievement inequality by estimating the degree to which SES gaps in achievement observed in school-age are already determined before children actually enter primary schooling. At the same time, we will empirically assess the importance of SES-related mechanisms shaping achievement gaps after children entered proper school life. In particular, we address three interrelated research questions: (1) How large are SES gaps in educational achievements before children enter school and how do these gaps develop during primary schooling? (2) To what extent are SES gaps in primary schooling attributable to SES gaps which are already settled before primary schooling? (3) Do answers to questions (1) and (2) relate to differences in the institutional context? We focus on achievement related to language, which is one of the most critical dimensions of cognitive 
development in the early years of life and crucial for educational success and later life chances (Alexander, 2008; Farkas \& Beron, 2004).

A previous study by Bradbury and colleagues (2015) suggests that a great part of SES inequality in achievement at the end of primary schooling is rooted in the preschool-age period in anglophone and liberal countries, such as the UK, the US, and Australia (Bradbury et al., 2015). However, cross-national evidence, possibly including European countries characterized by different institutional settings, is needed to assess the generalizability of these earlier findings. We address this gap by replicating results of Bradbury and colleagues (2015) for the UK and adding new evidence on Germany and the Netherlands, two national contexts not examined so far. Germany and the Netherlands are theoretically interesting cases as they contrast in many institutional and sociocultural ways the typical liberal welfare state context of anglophone countries previously studied. Linking these cases with the case of the UK does not only provide an intriguing comparative setting but also ensures comparability to previous research and findings.

\section{Theoretical framework}

The dynamic nature of skill formation

Understanding why there are socioeconomic inequalities in achievement among children in school age requires insights on the process through which such differences are generated starting from early childhood. Skill formation is often described as a process through which skills - rather than simply inherited - are dynamically produced starting from the early years. Cunha and Heckman $(2007,2008)$ describe the process of skill formation as a multistage and dynamic process in which the current skill level $\left(\varphi_{t}\right)$ is a positive function of previously acquired skills $\left(\varphi_{t-1}\right)$ and past family environment and investment $\left(I_{t-1}\right)$ :

$$
\varphi_{t}=f\left(\varphi_{t-1}, I_{t-1}\right)
$$

The recent models of skill formation convey two important ideas regarding skill development at the individual level. First and foremost, skills are self-productive because the future acquisition of skills $\left(\varphi_{t}\right)$ is higher for higher stocks of previously accumulated skills $\left(\varphi_{t-1}\right)$. This dynamic leads to path-dependencies in the sense that 'skill begets skill'. Second, past family environment and investment $\left(I_{t-1}\right)$ are not only effective in the short-run $\left(\varphi_{t}\right)$ but bear 
also long-lasting consequences due the indirect influence they have on subsequent skill levels (for example, $\left.\varphi_{t+2}\right)$ through the development of skills in between $\left(\varphi_{t}\right.$ and $\left.\varphi_{t+1}\right)$. Here, we focus on the dynamics of skill formation between two phases of children's life courses: the preschool period - ranging from birth up to pre-primary education - and the school period ranging from the school start towards the end of primary school.

Parental background shapes the family environment and investments into children's development, thus generating social inequalities in skill development even when there are no innate differences in intelligence. In line with this premise, social causation models connect parental SES and child outcomes through a variety of mechanisms, most of which relate to the quality of parent-child interactions and the financial resources. High SES families avail of better material and immaterial resources to cope with children's biological and emotional needs and are less likely to experience economic hardship, both of which reflects in better cognitive development (Conger \& Donnellan, 2007; McLoyd, 1998). For example, highly educated parents may involve their children in educationally-relevant activities (Ermisch, 2008; Lareau, 2006), and are less likely to experience financial constraints that strain routine parenting practices (Dotterer, Iheoma, \& Pungello, 2012; Hoff, Laursen, \& Tardif, 2002).

In the early years of life parental investment and family environment are particularly consequential for cognitive development. Indeed, children's brain is malleable and most receptive to external stimuli at the early stages (Doyle, Harmon, Heckman, \& Tremblay, 2009; Sowell et al., 2003). Hence, short and long-run effects of SES differentials in family environment and investment operating from birth up to the school entry should contribute to the emergence of SES inequality in children's educational achievements already before the start of school life.

The model of skill formation also speaks for a certain resilience of SES-achievement inequality over time. According to the principle of self-productivity of skills at the individual level, if current skills are a positive function of past skills, then current disparities among children will be persistent throughout future achievement distributions. Even in absence of SES differences in early achievements, the distributional rank at school entry will determine the distributional rank at later educational stages, at least to some extent. As a consequence of such persistency in achievement ranks, it is likely that a certain fraction of SES inequality in achievement settled at the end of the preschool phase is carried over to primary schooling. Moreover, ability sorting in school systems may exacerbate further the effects of skill selfproductivity inducing persistency in SES-inequality in educational achievement. For example, children with better early educational achievement may be channeled into schools with higher 
quality teachers (Borman et al. 2005) or benefit from peer effects when matched into homogeneous classrooms or ability groups (Hoxby 2000; Gamoran 1992; Condron 2008).

In addition to persistency in achievement inequality, parental investment and family environments continue to shape children's educational experiences and learning opportunities after they transited to school life. School and classroom segregation along socioeconomic lines may foster unequal learning opportunities for children who actually started into school life with similar intellectual resources. Hence, we would expect that SES is associated with school age achievement over and above achievements settled before school entry. For example, higher SES families may be more efficient in detecting and compensating for a poor start of their children or even try to secure further advantages after a bright start (Bernardi, 2014; Passaretta $\&$ Skopek, 2018). The mechanisms working towards the compensation for a poor-performing start or the boosting of a high-performing start relate to various forms of parental investment and family environment ranging from parents' direct involvement - for example, via stimulation or emotional support at home - to the exploitation of opportunities offered by primary school differentiation - for example, via enrolment in high-quality schools, private education, or private tutoring. A related question is whether there is symmetry between compensatory of boosting dynamics or, to the contrary, whether there is asymmetry in which either of the two dominates. In the latter case, the impact of SES on children's school achievement will be a function of their earlier (pre-school) achievement.

\section{Analytical framework and objectives}

To link those theoretical considerations with our empirical setup, we draw upon an analytical mediation framework that distinguishes two main channels through which socioeconomic status operates on language outcomes of school-aged children (see Figure 1). An indirect channel, which runs through opportunities of early cognitive development and language acquisition from infancy to preschool age up to the school start. And a direct channel, which operates via the additional role of social background unfolding over schooling. The indirect channel requires that parental SES plays a role before the school enrolment $(A)$ and that skill development is a dynamic process in which earlier skill development predicts later skill outcomes $(B)$. Hence, the indirect channel (path $A B$ ) captures mechanisms of social inequality in achievement that operate mostly before the exposure to formal teaching but are consequential for the social inequality in the classroom. The direct channel requires that, over and above a child's preschool-age achievement, parental SES relates to additional 
(dis)advantage in development and learning $(C)$. Hence, the direct channel captures mechanisms of social inequality unfolding during school age, i.e. after children had entered formal schooling. Finally, the dashed line indicates that the direct channel might be a function of prior achievement which results in a more complex scenario of moderated mediation. For example, if there is more compensation at the bottom and less boosting at the top, the direct channel of SES will be larger at the lower end and smaller at the upper end of the preschoolage achievement distribution.

\section{$===$ FIGURE $1==$}

Our primary goal is to assess the relative strength of the two analytical channels, which mirror the overall outcome of the various mechanisms of social inequality operating before (indirect) and during (direct) school life. Empirical evidence on the relative strength of these channels is not only of academic use but also bears important practical implications for social policy making. If most of SES inequality in school-age is attributable to the indirect channel, then the most effective way of reducing social inequality in learning during the school years is via interventions combatting social inequality early in life, virtually prior to school enrolment. Note that such conclusions cannot be reached via the simple description of the evolution of SES inequality in achievement from early childhood to school life.

It is beyond the scope of the paper to test the complex array of specific micromechanisms which may be causally constitutive to the SES-achievement gap. Nonetheless, our analysis provides an overarching framework to research focusing on specific inequality mechanisms by stressing the importance of two distinct phases - preschool and school age in the formation of social inequality in educational opportunities. In the following, we discuss the main cross-country institutional differences that may affect the strength of the links depicted in Figure 1.

\section{The institutional contexts}

\section{The three countries}

The UK resembles the 'liberal' welfare state regime that combines a residual role of the state in the societal sphere with comparatively high returns to education, which results in high inequality in the living conditions of families with different educational resources. Conversely, 
the more generous welfare states in Germany and the Netherlands compress living standards, for example, via direct transfers to families or educational support to educationally and economically disadvantaged parents.

In all countries, children in the early years (0-3) can avail of early education and care arrangements (hereafter: ECEC), while in the following years they usually attend Kindergarten (3-4 to 6). The organization and the attendance rates of ECEC and Kindergarten differ starkly across countries, however. In the UK, parents must rely on costly and scarcely available private ECEC services frequently run by low qualified staff, while being offered just free part-time care for children aged 3-4 (West, Blome, \& Lewis, 2019). Pre-primary education in England and Wales happens in the form of a 'reception class' at around age 4-5 which takes place before the first year of compulsory education ('Year One') but is still formally part of primary schooling. The UK system contrasts sharply with the Dutch system combining various ECEC arrangements with relatively high attendance rates (but low intensity) for children below age 4 and universal Kindergarten that starts when the child turn 4 years old (Leseman et al., 2017). Interestingly, Kindergarten is integrated with the primary school system in the Netherlands. A Dutch peculiarity are nation-wide programs that aim at reducing early educational and developmental disparities (e.g., the Dutch Educational Disadvantage Policy Onderwijsachterstandenbeleid). In between those extremes stands Germany, with a combination of a semi-standardized, scarcely subsidized ECEC sector and a standardized and nearly universal Kindergarten system (Blossfeld, Kulic, Skopek, \& Triventi, 2017). In contrast to the Netherlands, Germany has considerably less emphasis on targeted policies designed to reduce early inequalities.

Despite specific country differences in the organization and the timing of ECEC and Kindergarten, children in all countries are enrolled in some form of pre-primary education (ISCED 0) at around age 5, while starting primary schooling in the form of ISCED 1 only at around age 6-7. Hence, even though the Dutch Kindergarten and the British reception class are formally part of the primary school systems, formal teaching in the classroom (ISCED 1) starts at approximately the same age in all countries. In the remaining of the text, we refer to the preschool-age period as the period ranging from birth up to age 5, which is a biographical point in which children have not yet been exposed to formal teaching in primary school (ISCED 1) in all countries.

Primary school is comprehensive in all countries. Yet, there are some notable differences among school systems. Despite federal heterogeneity, primary education in Germany is highly standardized in terms of curricula, organisational structures, and teachers' 
education (Allmendinger, 1989). After Grade 4 (around age of 10), students enter the tracked secondary school system in most of the German states. In the UK, educational frameworks differ between the constituent countries. For example, England has a quasi-market of schools, with a strong emphasis on parental choice and school autonomy. In Scotland, however, school autonomy is more limited resulting in less competition between schools (for a more extensive discussion, see West et al. 2010). In contrast to Germany, UK applies no formal tracking in secondary school, yet, there is heterogeneity across types of secondary schools, curriculum differentiation by subject choice, and overall school quality. As in England, the Dutch primary education system is highly decentralized, and schools have a large degree of autonomy. This autonomy is balanced by a strong role of the inspectorate of education, however (OECD 2016). The national funding rules apply equally to all schools (both public and private) and schools with a higher share of disadvantaged children are allocated substantially more funding from the national education budget. However, school segregation is significant and increasing in the Netherlands (Ladd, Fiske, \& Ruijs, 2011). Like their German counterparts, Dutch students are channelled to secondary school tracks (at around age 12).

\section{Expectations about cross-country variations}

We expect to detect evidence for both the direct and the indirect channels in all three countries. The aforementioned institutional differences motivate some more nuanced hypotheses, however. First, we expect that Path $A$ (Figure 1) to be strongest in the UK. The comparably higher level of economic inequality might magnify SES inequality in the early years of life. At the same time, a relatively low utilization of ECEC in UK makes it unlikely that the larger inequality in family resources can be compensated, which, taken together, should translate into comparably large SES inequality in preschool-age achievement of UK children. Conversely, the stronger role of ECEC and Kindergarten in the Netherlands and Germany may compensate for the mechanisms of SES inequality unfolding over the early years. This might be true for Netherlands due to the targeted approach in the Dutch ECEC system. Summing up, we expect SES inequalities in preschool-age achievement to be smallest in the Netherlands, highest in the UK, and medium-level in Germany.

Second, as self-productivity of skills is a general principle, there is little a priori reason to expect cross-country differences in the dynamics of achievement from preschool to school age (Path $B$ in Figure 1). Moreover, as ability sorting is uncommon in the primary education sectors of all three countries, there is only very limited room for ability effects due to 
performance-based segregation across instructional environments. For these reasons, we expect to find rather similar effect sizes for Path B across countries.

Third, in relation to the direct channel of SES (Path $C$ in Figure 1), we expect to find the largest effect sizes for UK. The reasons behind this hypothesis are similar to those stated in the case of the formation of early SES-based inequality in achievement. A comparably larger inequality in economic living standards in the UK might magnify the role of mechanisms favoring the cumulative influence of SES on educational achievement over school age. Compared to UK's liberal, means-tested welfare state model, the more generous German and Dutch welfare systems may do better in limiting cumulative SES effects unfolding over the school years. Altogether, we expect to find the largest direct influence of SES on school-age achievement in the UK.

\section{Data and methods}

\section{Data and sample selection}

Our analysis draws on longitudinal data from the National Educational Panel Study in Germany (NEPS), the Cohort Study on Educational Careers in the Netherlands (COOL), and the Millennium Cohort Study in the UK (MCS). NEPS and COOL data have a multi-cohort sequence design in which children are followed longitudinally starting from different stages in their lives. We use the Kindergarten Cohort of the NEPS and the Cohort 1 of the COOL, which collected longitudinal information on samples of children around age 5 enrolled in Kindergarten in the years in 2010/2011 and 2007/2008 respectively up the end of primary school. The MCS is a birth-cohort study that collected longitudinal information on one single cohort of children born in the UK in 2000/2001 and followed up until 15 years of age (Hansen 2014). For the sake of comparability, we limit the analyses between age 5 - when most of the children are not yet exposed to formal teaching in primary school - and age 11 - the end of primary education approximately. While there are minor differences in the historical periods of the data collection in the three datasets, it is unlikely that cohort effects bias our crossnational comparisons significantly: all children are observed for the first time between 2005/2006 and 2010/2011. ${ }^{1}$

\footnotetext{
${ }^{1}$ We follow the competence development of children aged 5 in 2005/2006 in the UK, 2007/2008 in the Netherlands, and 2010/2011 in Germany.
} 
All child-cohort studies are representative at the national level ${ }^{2}$ and contain detailed information on children's language abilities and their parental background. The MCS, NEPS and COOL data include an overall sample of 19243, 2996, and 7075 children, respectively. From these samples, we removed children with no details on parental SES and migration background. From the NEPS and COOL samples, we additionally removed first generation migrants because they are excluded by design in the MCS. Furthermore, we used balanced samples by including children only for whom we have data on all waves in the observation window. The balanced analytical samples include 9866 children in the UK, 420 in Germany, and 1115 in the Netherlands.

Attrition in NEPS occurred mostly in the transition from kindergarten to grade 1 as a result of the school-based sampling design (only a subset of the Kindergarten sample attended schools that were sampled in following rounds, for documentation, see (Würbach, 2019). Hence, although not in a socially selective fashion, the sample size dropped substantially after the transition to primary education. In COOL, attrition is mainly a result of school dropouts after first and second waves. In both NEPS and COOL dropout risks are lifted if children switch school or are retained in a grade. These issues are less severe in the MCS due to child-based sampling design but there is still attrition over waves.

Attrition can induce bias if it is selective on the process under study, that is in our case, a function of achievement and SES. Hence, we tackled selective attrition via inverse probability weighting that is a commonly used method to correct bias in longitudinal data (Seaman \& White, 2013). Experimenting with multiple imputation as an alternative strategy to tackle attrition yielded very similar findings. The weighting strategy differed slightly across countries due to survey-specific circumstances (in the MCS, inverse probability weighting was combined with multiple imputation). Details on the procedure used to account for selective attrition are in Appendix A1.

\section{Language achievement}

We focus on language skills for theoretical and pragmatic reasons. First, language is a crucial cognitive domain in the early life course as language and the knowledge of vocabulary are

\footnotetext{
${ }^{2}$ The sampling design of the MCS differs slightly from NEPS and COOL. The MCS has an age-based design while NEPS and COOL have a school-based design. However, kindergarten is almost universal in Germany and mandatory by age 5 in the Netherlands, thus making children sampled in kindergarten representative of the population of children at the national level.
} 
necessary to communicate effectively with teachers and peers (Dockrell, Lindsay, \& Palikara, 2011 ) and mediate learning in a variety of competence domains, such as reading, writing and even mathematics (Mercer, Sams, Mercer, \& Sams, 2008; Shaftel et al., 2010; Snow et al., 2009). Second, language skills have substantial returns in the labor market (e.g., Hanushek, Schwerdt, Wiederhold, \& Woessmann, 2015) and evidence on the persistence of language gaps is therefore relevant for our understanding of intergenerational social mobility. Third, our focus on language enables the cross-national comparison as the national datasets used include test score data on language development measured consistently before ISCED 1 starts and during primary schooling.

Table 1 reports details on the nature and timing of language-related tests administered to children in each country and wave. In the MCS, language-related skills were measured by standard tests based on the British Ability Scales (BAS) in terms of expressive language abilities (age 5), reading abilities (age 7), and verbal reasoning and knowledge (age 11). NEPS used an adjusted version of the Peabody Picture Vocabulary Test (PPVT) to measure children's receptive vocabulary. Similar to NEPS, COOL tested receptive vocabulary/emergent literacy (age 5) and vocabulary knowledge (age 8 and 11). ${ }^{3}$ Although there are differences in timing and type of tests, all tests aim to measure language-related abilities and cover a similar observation window in the three countries.

Following standards in the literature (e.g., Bradbury et al. 2015; Feinstein 2003), we measure relative (z-scores) rather than absolute test score differences. Relative measures express gaps in relation to the overall variation of test scores and are, therefore, useful for gauging inequality in achievement across groups (Passaretta \& Skopek, 2018; Reardon, 2008). ${ }^{4}$ We z-standardized test scores within each wave to have a mean of 0 and a standard deviation of 1 , separately for each country. The strategy improves the comparability of achievement gaps across life stages within countries. Comparability between countries is more limited and hinges on several assumptions that we cannot test with our data, such as similar reliability and validity between different types of tests (e.g., the British Ability Scales versus the German adaption of the PPVT). This caveat should be kept in mind when comparing achievement gaps between countries. To increase precision of our estimation, we adjusted z-standardized test scores for slight age differences among children in the same life stage. Following Bradbury and

\footnotetext{
${ }^{3} \mathrm{COOL}$ also includes test data on reading skills (age 8 and 11). We obtain similar results when using data on reading instead of vocabulary scores.

${ }^{4}$ Strengths and limitations of the relative approach have been discussed extensively in other studies (see, for example, Skopek and Passaretta 2018).
} 
colleagues (2015), the procedure involved a $\mathrm{z}$-standardization of the residuals from a linear regression of raw test scores on a cubic function of age at test day (see Appendix A2 for details).

\section{$===$ TABLE $1==$}

\section{Covariates}

Similar to previous studies on SES-achievement gaps (e.g., Bradbury et al 2015; Linberg et al. 2019), we used parental education as a proxy for parental SES. Although not covering all facets of socioeconomic status (Duncan, Magnuson, \& Votruba-Drzal, 2015), parental education is one of the most dominant and stable factors determining the socioeconomic position of a family. We measured parental education by the highest years of full-time education among parents. In addition to the metric variable, we also used a categorical version of the variable by distinguishing low (12 years or less), medium (12 to 14), and high (15 or more) educated parents. ${ }^{5}$ The metric and the group-based approach to the measurement of parental education are complementary. On the one hand, a single metric allows parsimonious models which brings advantages for working with the relatively small samples of COOL and NEPS. On the other hand, categorical variables enable a better examination of potential nonlinear relationships between parental education and children's achievement. In addition, a group-based approach might be more relevant when considering targeted policy interventions. Small samples prevented us from estimating the separate associations of different SES dimensions, such as parental income or occupation. However, additional analyses using household income (whenever possible) as the primary SES indicator provided very similar results. ${ }^{6}$

For all analyses we control for migration background, which is a separate dimension shaping achievement inequality but correlated with SES (Passaretta \& Skopek, 2018). Migration background is defined by having at least one parent not born in the country. Note that, for our purpose, we did not control for mediating variables that reflect specific social inequality mechanisms - such as parenting styles, school choice, or family demographic

\footnotetext{
${ }^{5}$ We defined the groups using other thresholds according to country-specific characteristics of the education systems but obtained very similar results to the one presented in the manuscript.

${ }^{6}$ In Germany and the Netherlands years of education reflect the years that are necessary to acquire the highest education level among the parents. In the UK, the indicator reflects the actual years spent in full-time education. This difference in measurements may slightly underestimate SES gaps in the UK compared to Germany and the Netherlands.
} 
characteristics - nor factors that are uncorrelated with SES in a representative sample - such as gender.

Analytical strategy

We estimated three main sets of models for each country. The first set estimates the overall SES gap in achievement $\left(\alpha_{1 t}\right)$ at each time point (wave $t$ ) adjusted for migration background (MIG):

$$
\begin{gathered}
z_{i t}=\alpha_{0 t}+\alpha_{1 t} \mathrm{SES}_{i}+\alpha_{2 t} \mathrm{MIG}_{i}+\epsilon_{i t} \\
\text { for } t \in\{1,2,3\}
\end{gathered}
$$

where $z_{i t}$ refers to the z-score of the child $i$ at time point $t$. A second set estimates the separate effects of the initial achievement, measured at the end of the preschool-age period $(t=1)$, and SES on later achievement in school age $(t=2$ and $t=3)$ :

$$
\begin{gathered}
z_{i t}=\beta_{0 t}+\beta_{1 t} \mathrm{SES}_{i}+\beta_{2 t} \mathrm{MIG}_{i}+\beta_{3 t} z_{i 1}+\epsilon_{i t} \\
\text { for } t \in\{2,3\}
\end{gathered}
$$

Parameter $\beta_{3 t}$ models the conditional persistence of achievement hierarchies over time in the three countries. Note that parameter $\beta_{1 t}$ identifies the additional, direct influence of SES on school-age achievement that comes on top of inequality observed before the school period began. The ratio between parameters $\beta_{1 t}$ and $\alpha_{1 t}$ - the direct and the overall SES effects indicates the relative importance of the direct (and implicitly the indirect) path leading to SES inequality in primary school achievements.

In a third set of models, we allow for an interaction between SES and initial achievement. Equation 3 specifies the direct SES association to be a function of achievement measured in the end of preschool age:

$$
\begin{gathered}
z_{i t}=\gamma_{0 t}+\gamma_{1 t} \mathrm{SES}_{i}+\gamma_{2 t} \mathrm{MIG}_{i}+\gamma_{3 t} z_{i 1}+\gamma_{4 t} z_{i 1} \mathrm{SES}_{i}+\epsilon_{i t} \\
\text { for } t \in\{2,3\}
\end{gathered}
$$

By this model we aimed to test our theoretical ideas on SES acting mostly as compensator for poor initial achievement or acting mostly as a booster allowing high initial achievers to gain 
further advantage from their parental background over the school years. In case compensation prevails, that is if $\gamma_{4 t}<0$, additional SES advantages would be stronger among initially low achieving children. Conversely, in case boosting prevails, i.e. if $\gamma_{4 t}>0$, additional SES advantages would be stronger on the upper end of the initial achievement distribution. If $\gamma_{4 t}=$ 0, Equation (3) reduces to (2) stating that additional (dis-)advantages related to SES are equally distributed over the distribution of preschool-age achievements (provided $\gamma_{1 t}>0$ ). Thus, estimating Equation (3) allows us to assess whether either compensation or boosting prevail or whether both dynamics have similar strength.

Equations (1) and (2) are estimated using parental education as both a metric and a group variable. For the sake of parsimony Equation (3) is estimated only based on the metric variable.

\section{Measurement error and regression to the mean}

The estimation of the conditional persistence in Equation (2) and (3) using standard OLS models invokes issues of endogeneity if there is measurement error in initial test scores $\left(z_{i 1}\right)$ as a measure of child achievement. Ignoring measurement error in test scores on the right-hand side of (2) and (3) may lead to artifactual estimates driven by regression-to-the-mean (hereafter: RTM). Past literature provides explicit discussions on RTM issues in relation to estimating achievement trajectories (Bynner et al., 2015; Jerrim \& Vignoles, 2013). In our case, ignoring measurement error in test scores provokes an underestimation of the conditional persistence in relative achievement (for example, $\beta_{3 t}$ in Equation 2) and an overestimation of the direct SES effect ( $\beta_{1 t}$ in Equation 2).

We counter measurement error in test scores by using an instrument variable (IV) approach that minimizes biases arising from measurement error in the preschool-age test (Bradbury et al., 2015; Jerrim \& Vignoles, 2013). The IV strategy involves using another preschool-age score to predict the actual preschool-age score of interest and, in a second stage, plug the predicted rather than the observed values of such score into Equations 2 and 3. Under the condition that the instrument is relevant, e.g., highly correlated with the instrumented variable, and that measurement error is independent between scores, these predictions yield error-free signals of preschool-age achievements. We satisfy these assumptions by using instruments which are highly correlated with language scores in preschool age - such as scientific literacy, vocabulary, and arithmetic - that are taken on a different day (NEPS, COOL) or even in a previous wave (in the case of MCS). The temporal distance between the instrument 
test and the focal test ensures the elimination of day specific sources of measurement error (e.g., having luck or bad luck on a day). Moreover, measurement error due to familiarity with particular test items is accounted for given that the exact test items in the primary test do not overlap with test items in the test used as an instrument. In all countries, we applied two-stage least square (2SLS) for the IV estimation. More details on our IV strategy including the exact specification of first- and second stages can be found in Appendix A3.

Even if we cannot rule out entirely other possible sources of measurement error, we are confident that the adopted IV strategy addresses the major issues. Nevertheless, we will interpret estimates on the conditional persistence and on the relative importance of the indirect channel as lower bound estimates and estimates for the residual SES effect - the direct channel - as upper bound estimates. Eventually, we will also compare results from our IV estimation with results from standard OLS estimation which assumes error-free achievement measures.

\section{Empirical results}

Overall SES gaps in achievement before and during school age

Table 2 and 3 report the total association (TOTAL) between parental SES and children's language-related skills before primary schooling (age 5) and over the school age (age 7-8 and 9-11) for the three countries. In line with the previous research, we find positive and statistically significant associations between parental SES and achievement in all countries and periods considered. Notably, SES inequality in achievement is already substantial when children are only 5 years of age, a point in time by which children are not yet in school in Germany and have just started school in the UK and the Netherlands. Overall, we find the social gradient at age 5 to be highest in Germany (see Table 2). Estimates suggest that each additional year of parental education in the German context is associated with roughly $12 \%$ of a standard deviation increase in language achievement $(b=0.120)$. Over the full range -8 years versus 18 years of highest parental education - the estimate implies a gap of 1.2 standard deviations. Conversely, gaps are the smallest in the Netherlands $(b=0.041)$. Gap estimates for the UK are in between $(b=0.105)$. The same cross-national pattern is found in the analyses using the categorical measurement of SES (see Table 3). The gaps between low SES children compared with medium and high SES children at age 5 are the highest in Germany, followed by the UK and the Netherlands respectively. This is observation is only partly in line with our theoretical expectations but consistent to a recent study that detected larger SES-achievements 
gaps in Germany when compared with the liberal context of the United States (Linberg, Schneider, Waldfogel, \& Wang, 2019). However, these numerical results should be interpreted with some caution since the exact underlying tests - even though related to the same cognitive domain - differed between countries and the statistical uncertainty (standard errors) are comparably large for the German case.

How does SES inequality in language achievement develop over the years of primary schooling? In the UK SES gaps remain constant throughout primary schooling, and this holds both when looking at the results from the metric and the group-based approach to the measurement of SES (effect size 10\% of a standard deviation for each additional year of parental education; $25-30 \%$ of a standard deviation comparing medium and low SES kids; 55 $60 \%$ of a standard deviation comparing high and low SES kids). In contrast, we observe increasing achievement gaps over schooling in Germany and the Netherlands. Comparing the first (age 5) with the last time point (ages 9 and 11 respectively), the social gradient increases from $12 \%$ to $15 \%$ of a standard deviation $(\mathrm{p}=.448)$ per year of parental education in Germany and more than doubles from about $4 \%$ to $10 \%$ in the Netherlands $(\mathrm{p}=.005)$. Similar patterns are found when looking at changes between SES groups. Taken together, the data at hand does not provide compelling evidence for systematically growing or shrinking gaps in Germany and the UK. However, there is some evidence for SES gaps in language achievement to increase in the Netherlands.

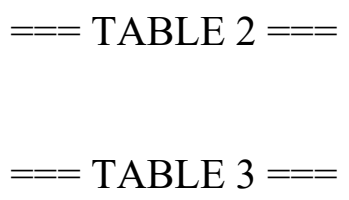

The role of preschool-age achievement and SES for school-age achievement

Column DIRECT in Tables 2 and 3 report estimates for the direct effect of SES on language achievement over the school age once achievement at age 5 is taken into account. We report both OLS and IV estimates. The latter estimates adjust for measurement error in age 5 scores. We comment first on the results obtained by measuring SES as a metric variable (Table 2) and then on the results from the group-based approach (Table 3).

Estimates in Table 2 reveal a strong and statistically significant association between preschool and school-age achievement in all countries. As the IV estimates suggest, achievement hierarchies settled before school entry tend to persist over primary schooling but, 
at the same time, there is still room for an additional role of SES during school-age. A comparison between OLS and IV estimates clearly supports our hunch that ignoring measurement error would severely underestimate persistency in relative achievement (e.g., .331 for OLS versus .579 for IV at Age 7 in the UK) and, as a result, overestimate the direct effect of SES on later achievement (e.g., .075 for OLS versus .049 for IV at Age 7 in the UK) in all countries. Thus, we continue to interpret only the results from the more reliable IV estimation.

Interestingly, the conditional persistence (coefficient for Age 5) is stronger in Germany (.86-.92) compared to the Netherlands (about .67) and the UK (.53-.58). These findings imply that, in terms of their relative achievement and conditional on their social background, children in the UK show the highest mobility while children in Germany show the lowest mobility over time. In contrast, the direct effect of SES on school-age achievement is rather similar in magnitude across all countries (in particular for Age 7). Note that direct effects fail to pass statistical test for the German case which is owed to the small sample size. In other words, over and above initial achievement, SES seems to play a role on children's relative achievement during primary school. Taken together, while these findings mostly support our general hypotheses regarding the role of earlier achievement and SES for later achievement, they do not support our hypotheses about the homogeneity in achievement persistence and the heterogeneity in the direct SES effect across countries.

Findings are substantively the same when re-running our models using group dummies for SES instead of the parental years of education (see Table 3). Here too standard OLS models would underestimate the conditional persistence while overestimating the residual SESachievement association. The findings from the metric and the group-based approach are very similar in the case of the UK and Germany, which supports the linearity assumption behind the metric measurement of SES. The categorical models, however, reveal that the residual SESachievement association is not linear in the case of the Netherlands. When controlling for measurement error (IV), a statistically significant residual SES effect remains only for the contrast between high and low SES children (and the contrast between high and medium SES children, not shown in the table). Taken together, only the Dutch data suggests some nonlinearity in the direct effect of SES to the extent that only children from parents at the top of the social hierarchy seem to benefit over proportionally from their social standing during school-age.

All the results from the first two sets of models suggest a clear conclusion: a substantial part of the SES inequality in school-age achievement is already determined the start of the 
school phase. Nonetheless, our models have estimated the average SES effect on achievement once initial performance is taken into account (Equation 2). However, the direct SES effect may vary depending on the initial level of performance (Equation 3). Does the additional SES (dis-)advantage concentrate at certain points of the distribution of early achievements? Does SES mainly compensate for a poor start in terms of achievement? Or, does parental SES mainly help children with a 'bright' start to further excel in their school life?

Figure 2 visualizes estimation results on the statistical interactions specified in Equation (3). The visual inspection allows us to gauge how the direct SES effect (for the two time points in school and for each country) may vary as a function of achievement at age 5. Negative slopes indicate stronger compensatory effects, positive slopes stronger boosting effects, and zeroslopes constant SES effects over the distribution of preschool-age achievements. Results from the British and German data suggest that advantages by parental SES concentrate at the low end of the achievement distribution at age $5 .^{7}$ On the flip side, among children who perform high by the end of the preschool phase, SES effects during schooling are weaker. Thus, SES seems to compensate for a poor start rather than boosting further a bright start. For the Netherlands, the Figure indicates the opposite result at age 11: SES makes a larger difference among the high performers in preschool age. However, interactions terms are statistically significant only in the UK and, therefore, it is questionable whether these findings apply beyond our samples in the Netherlands and Germany (see Table B5 in Appendix B). Even though the data shows some intriguing tendencies in terms of the prevalence of boosting or compensatory effects of SES, we have limited support for the prevalence of either the two dynamics in the two non-Anglo-Saxon countries, possibly due to small simple size. Therefore, we conclude that, by and large, the direct SES effect is homogenous among high and low performing children in preschool age in Germany and the Netherlands. Conversely, a compensatory advantage seems to prevail over boosting in the UK.

$===$ FIGURE $2==$

\footnotetext{
${ }^{7}$ For example in the case of the UK, among children whose language performance at age 5 scores one standard deviation below the average ( -1 on the $\mathrm{X}$-axis), the gap at age 11 is estimated at $6 \%$ of a standard deviation per year of parental education; that corresponds to a full range gap (18 vs 8 years of parental education) of $60 \%$ of standard deviation among children who initially started at the same low performance level.
} 
Summary: To what extent is social inequality in school-age achievement determined before and during primary schooling?

Based on the model specified by Equation (2), Table 4 summarizes the relative importance of the direct ( $\mathrm{C}$ in Figure 1) and indirect channel (AB in Figure 1) relating SES to school age achievement. The Table shows the percentage of the SES inequality in school age that is explained by the amount of SES inequality accumulated by age 5 (indirect channel AB). Note that the percentage explained by the direct channel is simply 100 minus the percentage explained by the indirect channel. Numbers are shown separately for each country, time-point over primary schooling, and measurement approach to SES (metric versus group-based). In addition, we present the results from both standard OLS and the two-step IV estimation.

Our IV estimates let us conclude that a lion share of the achievement gaps observed in school-age are determined before children enter school life in Germany and the UK. As the IV estimates suggest (see Table 2 and 3), unequal achievement at age 5 explains from 54 to 63 per cent of the overall SES gaps observed in school age in the UK (a minimum of 54 per cent at age 7 comparing high with low SES kids - a fraction of $1-.292 / .636=.55-$ and a maximum of 63 at age 11 comparing medium with low SES kids - a fraction of $1-.092 / .248=0.63$ ). These shares are even higher in Germany, where preschool age inequality is responsible for 67 to 83 per cent of school-age gaps. Social inequality in preschool age is not the whole story of social inequality in school, though. Our estimates suggest that from 17 to 46 per cent of the SES-achievement link in school is attributable to mechanisms that operate after children entered school life in UK and Germany.

In the Netherlands we observe a partly different scenario. Based on the IV estimates from the metric approach, one would conclude that language differences observed in kindergarten age explain the minority of social inequalities in school age (around $40 \%$ and $30 \%$ at age 8 and 11, respectively). However, when resorting to the comparison between SES groups, some interesting differences emerge. Consistently with the results found in the UK and especially Germany, language differences observed in kindergarten age explain around 62$81 \%$ of later gaps between medium and low SES children. The scenario is very different when looking at the high-low SES gap in school age, which seems to be driven by language inequality observed in preschool age to a lower extent (33-47\%). While the majority of the high-low gap during primary schooling may be explained by the additional role that socioeconomic background may play beyond kindergarten in the Dutch context, we need to bear in mind that our estimation may exaggerate the importance of the direct channel. 
Finally, we want to highlight that standard OLS models that ignore measurement error produce a highly biased picture. As Table 4 demonstrates, naïve OLS models severely underestimate the relative importance of the indirect channel in comparison to IV estimation. Taking an average among all countries, time-points and SES-groups comparisons, we conclude that the proportion of SES inequality in school-age achievement explained by SES inequality in preschool is $40 \%$ higher when we correct for measurement error. As a result, OLS models overestimate the importance of the direct channel to a great extent. If measurement error responsible for RTM was neglected, one would mistakenly conclude that the great majority of SES inequality in achievement among school-aged children (from half to even $83 \%$ ) is generated during the school period in all countries.

All in all, our findings underline that social inequality in school-age achievement is to a good deal a persistent phenomenon which roots early in children's lives. Hence, turning more attention to the various bio-psycho-social mechanisms that link parental SES to child achievement in early childhood seems imperative for educational sociologists who want to understand inequality of educational opportunity in the school system.

\section{$===$ TABLE $4===$}

\section{Conclusions}

Drawing upon child cohort data from Germany, the Netherlands, and the UK, we estimated SES gaps in educational achievement and the extent to which SES gaps in school-age are determined by SES gaps settled before the school entry. We traced children's relative achievement in language-related skills at age 5, when children are usually enrolled in preprimary education, and at later ages during mid and end of the primary schooling phase.

Consistent to previous research on child development, we first found that SES inequality in language achievement are well-established even before children had entered formal schooling. Although some caution is needed when comparing test metrics between countries, our data suggested that early SES gaps were largest in Germany followed by the UK and, last, by the Netherlands, which features an ECEC system that integrates targeted programs for children from educationally disadvantaged backgrounds. Second, in relative terms, the degree of SES inequality in language skills remained fairly stable over primary schooling in the UK and Germany but was increasing in the Netherlands. At end of primary schooling levels of SES inequality in achievement were rather similar across countries, however. 
Third, we found evidence for substantial persistency in SES inequality in achievement ranks over time. Our analyses suggest that a major share of SES inequalities in school-age achievements was explained by the SES inequality settled by the end of the preschool phase. The 'indirect' channel was responsible for around 55-60\% of the school-age gap in the UK, and even up to $60-80 \%$ in Germany and the Netherlands (for the latter this holds only when looking at the gap between medium and low SES kids). This general finding is consistent with a previous study by Bradbury and colleagues (2015), which suggested that around $60-70 \%$ of SES inequality in achievement at the end of primary schooling is rooted in the preschool-age period in anglophone countries. We suggest that this proportion might even be higher in countries characterized by different institutional contexts, such as Germany and the Netherlands. Hence, the majority of the frequently reported social gaps in educational achievement among school-aged children have to be explained by inequality mechanisms operating in children's early years rather than the school years.

Our results show also that a non-negligible proportion of SES gaps in school age may be attributable to factors related to social background that continue to shape children's achievement over schooling. We estimated that the direct channel was responsible for around $20-40 \%$ (Germany and the Netherlands) to $40-45 \%$ (the UK) of the SES-achievement link at mid and end of primary schooling. An exception to these general patterns is the language advantage over primary schooling of Dutch children from high parental education, which seems to be mainly a result of mechanisms operating during the school years.

Our study contributes to research in sociology of education by drawing scholars' attention to the importance of those mechanisms of (dis-)advantage which generate unequal outcomes in school life but operate long before children enter the classroom. Our study also invites further research to extend theoretical models and empirical specifications of educational (dis-)advantage by dynamic aspects of educational inequality. Furthermore, our findings were revealed only once we were accounting for measurement error in underlying test scores. Thus, an important methodological message from our study is that a naïve estimation that assume error free achievement measures would underestimate the dynamics of achievement inequality. Had we relied on standard OLS estimation, our conclusions would have been opposite, namely, that most of social inequality in achievement is produced by mechanisms of social inequality operating during schooling.

Our results bear also relevant implications for social policy making. First of all, promoting more equal opportunities before children go to school seems to be the most effective way of reducing social inequalities in school-age achievement. In this regard, our findings 
connect to a large body of research arguing that early educational programs can yield substantial benefits particularly for disadvantaged children (see Kulic, Skopek, Triventi, \& Blossfeld, 2019; van Huizen \& Plantenga, 2018). Hence, whether directed towards the expansion of early education and care or the direct support within the family environment, early interventions have the potential to reduce not only social inequality in early educational achievement but also at later educational stages through positive dynamics in learning and achievement.

However, it seems that some social inequalities would show up along primary schooling even in the absence of social inequality before school exposure. We found traces of potential compensatory advantage in all three countries, although German results cannot be generalized beyond our sample. Hence, our results tentatively suggest that the parental socioeconomic status might compensate for initially poor performances. Moreover, compensatory dynamics seem to prevail over boosting's in the UK and there are hints this might be the case also in Germany. Conversely, we found signs of stronger boosting effects in the Netherlands: children from better-off families seem more likely to excel further when starting school with high intellectual capacity rather than climbing up the ladder after a poor start. But again, this latter subtle dynamic found no evidence beyond our sample. This discussion suggests that a second but less effective way of reducing social inequalities in school age is to minimize social inequality mechanisms enabling children from better-off families to take further advantage of their social background during the educational career. The latter goal can be achieved, for example, by reducing the SES-based sorting into schools or the informal differentiation between and within primary schools.

The cross-country comparison suggests that preschool-age interventions, while generally more effective than school-age interventions everywhere, would be particularly effective in Germany, where preschool-age gaps explain the vast majority of social inequality in achievement later on. A combination of preschool- and school-age interventions is desirable in the UK and the Netherlands, where mechanism of social inequality operating during school life seem to have comparatively higher importance compared to Germany. School-age interventions would be particularly effective in the Netherlands if the aim is to abating language gaps at the end of primary school between children at the top of the social hierarchy and all the others.

Note that our study is limited in several ways inviting further research. First of all, as it was not the purpose of our study, we cannot say anything about the manifold and specific micro-mechanisms that explain the SES-achievement association. Our research asked about 
the relative importance of the concert of those mechanisms of social inequality operating before and during school age. In the same vein, we did not differentiate school- and out-of-school factors behind the influence of SES. Further research might try to isolate separate mechanisms while keeping in mind that those mechanisms working early in the life course are particularly consequential in shaping later achievement inequality.

Second, our analysis focused on achievement inequality by studying relative and not absolute differences in achievement. Therefore, our analysis is silent about the evolution of social inequalities in terms of actual proficiency, an issue typically addressed by psychological research. Given appropriate data, the adoption of an absolute perspective could complement our focus on differences relative to the distribution. For example, absolute scores measuring proficiency would allow us to inspect closely exacerbatory and compensatory dynamics (Matthew effects and learning plateaus) that should reflect in increasing and decreasing test score variance over time. An absolute perspective would also allow checking whether $\mathrm{z}$-score gaps conceal more subtle dynamics in absolute terms: a constant relative gap over time may indeed conceal increasing or decreasing gaps on the absolute level if there are no overtaking.

Third, even though we adopted a careful strategy to guard against regression to the mean, it is still possible that we were unable to rule out entirely measurement error in the earliest scores, and that regression to the mean operates between the second and the third timepoints. This caveat may explain the importance of the direct channel of social reproduction we found in the Netherlands with regards of the latest measurement (age 11). Hence, it seems crucial to remind that our findings on the importance of the indirect channel may still be underestimated, and the importance of the direct channel overestimated. Besides, our study is limited in terms of the actual causal relationship between earlier and later achievement. For example, based on our data we cannot rule out that both earlier and later (language) achievement are co-determined by an underlying factor such as a general intelligence or genetic endowment. In that case, our estimates of the link between earlier and later achievement would overestimate their actual causal interdependence. Provided suitable data further research could for example exploit repeated measures on equivalent test scales and apply dynamic panel models to estimate the causal link between earlier and later achievement.

Finally, although all tests we used were related to language, our analyses in the UK and the Netherlands draw from partly different tests over time, which could create some uncertainty in our estimates. Such differences might explain differences in findings across countries but are unlikely to explain general similarities we have detected. Moreover, our findings are 
restricted to the intellectual domain of language. Further research using other data might test if the same patterns hold for different achievement domains such as numeracy and math. 


\section{References}

Alexander, R. J. (2008). Towards Dialogic Teaching: Rethinking Class- room Talk. York: Dialogos.

Allmendinger, J. (1989). Educational systems and labor market outcomes. European Sociological Review, 5(3), 231-250.

Barone, C. (2006). Cultural capital, ambition and the explanation of inequalities in learning outcomes: A comparative analysis. Sociology, 40(6), 1039-1058.

Barone, C., \& Werfhorst, H. G. Van De. (2011). Education, cognitive skills and earnings in comparative perspective.

Baumert, J., Nagy, G., \& Lehmann, R. (2012). Cumulative Advantages and the Emergence of Social and Ethnic Inequality: Matthew Effects in Reading and Mathematics Development Within Elementary Schools? Child Development, 83(4), 1347-1367.

Bernardi, F. (2014). Compensatory advantage as a mechanism of educational inequality: A regression discontinuity based on month of birth. Sociology of Education, 87(2), 74-88.

Blossfeld, H.-P., Kulic, N., Skopek, J., \& Triventi, M. (2017). Childcare, early education and social inequality : an international perspective, 2017, eduLIFE lifelong learning.

Cheltenham: Edward Elgar Publishing.

Borman, G. D., Kimball, S. M., Borman, G. D., \& Kimball, S. M. (2005). Teacher Quality and Educational Equality : Do Teachers with Higher Standards - Based Teacher Quality and Educational Equality : Do Teachers with Higher Standards- Ratings Close Student Achievement Gaps ?, 106(1), 3-20.

Bradbury, B., Corak, M., Waldfogel, J., \& Washbrook, E. (2015). Too many children left behind. The U.S. achievement gap in comparative perspective. New York: Russel Sage Foundation.

Bynner, J., Feinstein, L., Jerrim, J., Vignoles, A., Goldstein, H., French, R., ... Lupton, R. (2015). Social class differences in early cognitive development. Longitudinal and Life Course Studies, (6), 331-376.

Cheadle, J. E. (2009). Educational Investment, Family Context, and Children's Math and Reading Growth from Kindergarten through the Third Grade. Sociology of Education, 81(1), 1-31.

Chen, Q., Gelman, A., Tracy, M., Norris, F. H., \& Galea, S. (2012). Weighting Adjustments for Panel Nonresponse, 1-23.

Condron, D. J. (2008). An Early Start Skill Grouping and Unequal Reading Gains in the 
Elementary Years.pdf. The Sociological Quarterly, 42(2), 363-394.

Conger, R. D., \& Donnellan, M. B. (2007). An Interactionist Perspective on the Socioeconomic Context of Human Development. Annual Review of Psychology, (58), $175-199$.

Cunha, F., \& Heckman, J. J. (2008). Formulating, Identifying and Estimating the Technology of Cognitive and Noncognitive Skill Formation. Journal of Human Resources, 43(4), $738-782$.

DiPrete, T. A., \& Eirich, G. M. (2006). Cumulative Advantage as a Mechanism for Inequality: A Review of Theoretical and Empirical Developments. Annual Review of Sociology, 32(1), 271-297.

Dockrell, J. E., Lindsay, G., \& Palikara, O. (2011). Explaining the academic achievement at school leaving for pupils with a history of language impairment : Previous academic achievement and literacy skills.

Dotterer, A. M., Iheoma, U. I., \& Pungello, E. P. (2012). Parenting, Race, and Socioeconomic Status: Links to School Readiness. Family Relations, (61), 657-70.

Doyle, O., Harmon, C. P., Heckman, J. J., \& Tremblay, R. E. (2009). Investing in early human development: timing and economic efficiency. Economics \& Human Biology, $7(1), 1-6$.

Duncan, G. J., Magnuson, K., \& Votruba-Drzal, E. (2015). Children and socioeconomic status. In Handbook of Child Psychology and Developmental Science: Ecological Settings and Processes (pp. 534-573).

Ermisch, J. (2008). Origins of Social Immobility and Inequality: Parenting and Early Child Development, (205), 62-71.

Farkas, G., \& Beron, K. (2004). S cience S ocial knowledge : differences by class and race q, $33,464-497$.

Feinstein, L. (2003). Inequality in the early cognitive development of British children in the 1970 cohort. Economica, 70(277), 73-97.

Gamoran, A. (1992). Is Ability Grouping Equitable? Educational Leadership, 50, 11-17.

Hanushek, E. A., Schwerdt, G., Wiederhold, S., \& Woessmann, L. (2015). Returns to skills around the world: Evidence from PIAAC. European Economic Review, 73, 103-130.

Heckman, J. J., \& Cunha, F. (2007). The Technology of Skill Formation. The American Economic Review, 97(2), 31-47.

Hippel, P. T. Von, \& Hamrock, C. (2019). Do Test Score Gaps Grow Before, During, or Between the School Years? Measurement Artifacts and What We Can Know in Spite of 
Them. Sociological Science, 6, 43-80.

Hoff, E., Laursen, B., \& Tardif, T. (2002). Socioeconomic Status and Parenting. In M. H.

Bornstein (Ed.), The Handbook of Parenting: Biology and Ecology of Parenting (Vol. 2, pp. 231-52). Mahwah, New Jersey: Lawrence Erlbaum.

Jackson, M. (2013). Determined to Succeed? Performance versus Choice in Educational Attainment. Stanford: Stanford University Press.

Jerrim, J., \& Vignoles, A. (2013). Social mobility, regression to the mean and the cognitive development of high ability children from disadvantaged homes. Journal of the Royal Statistical Society. Series A: Statistics in Society, 176(4), 887-906.

Kerckhoff, A. C., Raudenbush, S. W., \& Glennie, E. (2001). Education, Cognitive Skill , and Labor Force Outcomes. Sociology of Education, 74(1), 1-24.

Kulic, N., Skopek, J., Triventi, M., \& Blossfeld, H. (2019). Social Background and Children's Cognitive Skills: The Role of Early Childhood Education and Care in a Cross-National Perspective. Annual Review of Sociology, 45.

Ladd, H. F., Fiske, E. B., \& Ruijs, N. M. (2011). Does Parental Choice Foster Segregated Schools? Insights from the Netherlands. In M. Berends, M. Cannata, \& E. B. Goldring (Eds.), School Choice and School Improvement (pp. 233-253). Cambridge: : Harvard Education Publishing.

Lareau, A. (2006). Unequal Childhoods: Class, Race and Family Life. Berkeley: University of California Press.

Leseman, P., Mulder, H., Verhagen, J., Broekhuizen, M., van Schaik, S., \& Slot, P. (2017). Effectiveness of Dutch targeted preschool education policy for disadvantaged children: Evidence from the Pre-COOL study. In H. P. Blossfeld, N. Kulic, J. Skopek, \& M. Triventi (Eds.), Childcare, Early Education and Social Inequality - An International Perspective (pp. 173-193). Cheltenham, UK: Edward Elgar.

Linberg, T., Schneider, T., Waldfogel, J., \& Wang, Y. (2019). Socioeconomic status gaps in child cognitive development in Germany and the United States. Social Science Research, 79, 1-31.

Marks, G. N., Cresswell, J., \& Ainley, J. (2006). Explaining socioeconomic inequalities in student achievement: The role of home and school factors. Educational Research and Evaluation, 12(2), 105-128.

McLoyd, V. C. (1998). Socioeconomic Disadvantage and Child Development. American Psychologist, 53(2), 185-204.

Mercer, N., Sams, C., Mercer, N., \& Sams, C. (2008). Teaching Children How to Use 
Language to Solve Maths Problems Teaching Children How to Use Language to Solve Maths Problems, 0782.

Ministry of Education Culture and Science. (2016). Key Figures Education.

OECD. (2016). Netherlands 2016: Foundations for the Future. Reviews of National Policies for Education.

Passaretta, G., Barbieri, P., Wolbers, M. H. J., \& Visser, M. (2018). The direct effect of social origin on men's occupational attainment over the early life course: An ItalianDutch comparison. Research in Social Stratification and Mobility, 56(March), 1-11.

Passaretta, G., \& Skopek, J. (2018). Roots and Development of Achievement Gaps. A Longitudinal Assessment in Selected European Countries. Dublin.

Potter, D., \& Roksa, J. (2013). Accumulating advantages over time : Family experiences and social class inequality in academic achievement. Social Science Research, 42(4), 10181032.

Protopapas, A., Sideridis, G. D., Mouzaki, A., \& Panagiotis, S. G. (2011). Matthew Effects in Reading Comprehension: Myth or Reality? Journal of Learning Disabilities, 44(5), $402-420$.

Reardon, S. F. (2008). Thirteen Ways of Looking at the Black-White Test Score Gap (IREPP Working Paper No. 2008-08). IREPP Working Paper.

Seaman, S. R., \& White, I. R. (2013). Review of inverse probability weighting for dealing with missing data. Statistical Methods in Medical Research, 22(3), 278-295.

Shaftel, J., Belton-kocher, E., Glasnapp, D., Poggio, J., Shaftel, J., Belton-kocher, E., ... Belton-kocher, E. (2010). The Impact of Language Characteristics in Mathematics Test Items on the Performance of English Language Learners and Students With Disabilities The Impact of Language Characteristics in Mathematics Test Items on the Performance of English Language Learner, 7197.

Skopek, J., \& Passaretta, G. (2018). The Social Stratification of Skills from Infancy to Adolescence - Evidence from an Accelerated Longitudinal Design. SocArXiv Papers, 1-63. Retrieved from https://osf.io/preprints/socarxiv/xkctv/

Snow, C. E., Lawrence, J. F., White, C., Snow, C. E., Lawrence, J. F., Generating, C. W., ... White, C. (2009). Generating Knowledge of Academic Language Among Urban Middle School Students, 5747.

Sowell, E. R., Peterson, B. S., Thompson, P. M., Welcome, S. E., Henkenius, A. L., \& Toga, A. W. (2003). Mapping cortical change across the human life span. Nature Neuroscience, 309-315. 
van Huizen, T., \& Plantenga, J. (2018). Do Children Benefit from Universal Early Childhood Education and Care? A Meta-Analysis of Evidence from Natural Experiments. Economics of Education Review, (66), 206-222.

West, A., Allmendinger, J., Nikolai, R., \& Barham, E. (2010). Decentralisation and educational achievement in Germany and the UK. Environment and Planning C: Government and Policy, 28(3), 450-468.

West, A., Blome, A., \& Lewis, J. (2019). What Characteristics of Funding, Provision and Regulation Are Associated with Effective Social Investment in ECEC in England, France and Germany? Journal of Social Policy, 1-24.

Würbach, A. (2019). Samples, Weights and Nonresponse: the Kindergarten Cohort of the National Educational Panel Study. Bamberg, Germany. 


\section{Tables}

Table 1 Observation window and competence assessments for the three countries

\begin{tabular}{lllll}
\hline & Preschool & Grade 1 & Grade 3 & Grade 5/6 \\
& $\sim$ age 5 & $\sim$ age 7 & $\sim$ age 8/9 & $\sim$ age 11 \\
\hline United Kingdom & BAS Naming & BAS Word & & BAS Verbal \\
& Vocabulary & Reading & & Similarities \\
Netherlands & $\begin{array}{l}\text { Language/emergent } \\
\text { literacy }\end{array}$ & & Vocabulary & Vocabulary \\
Germany & Vocabulary & Vocabulary & Vocabulary & \\
\hline
\end{tabular}


Table 2 Total and direct effects of SES on achievement by age.

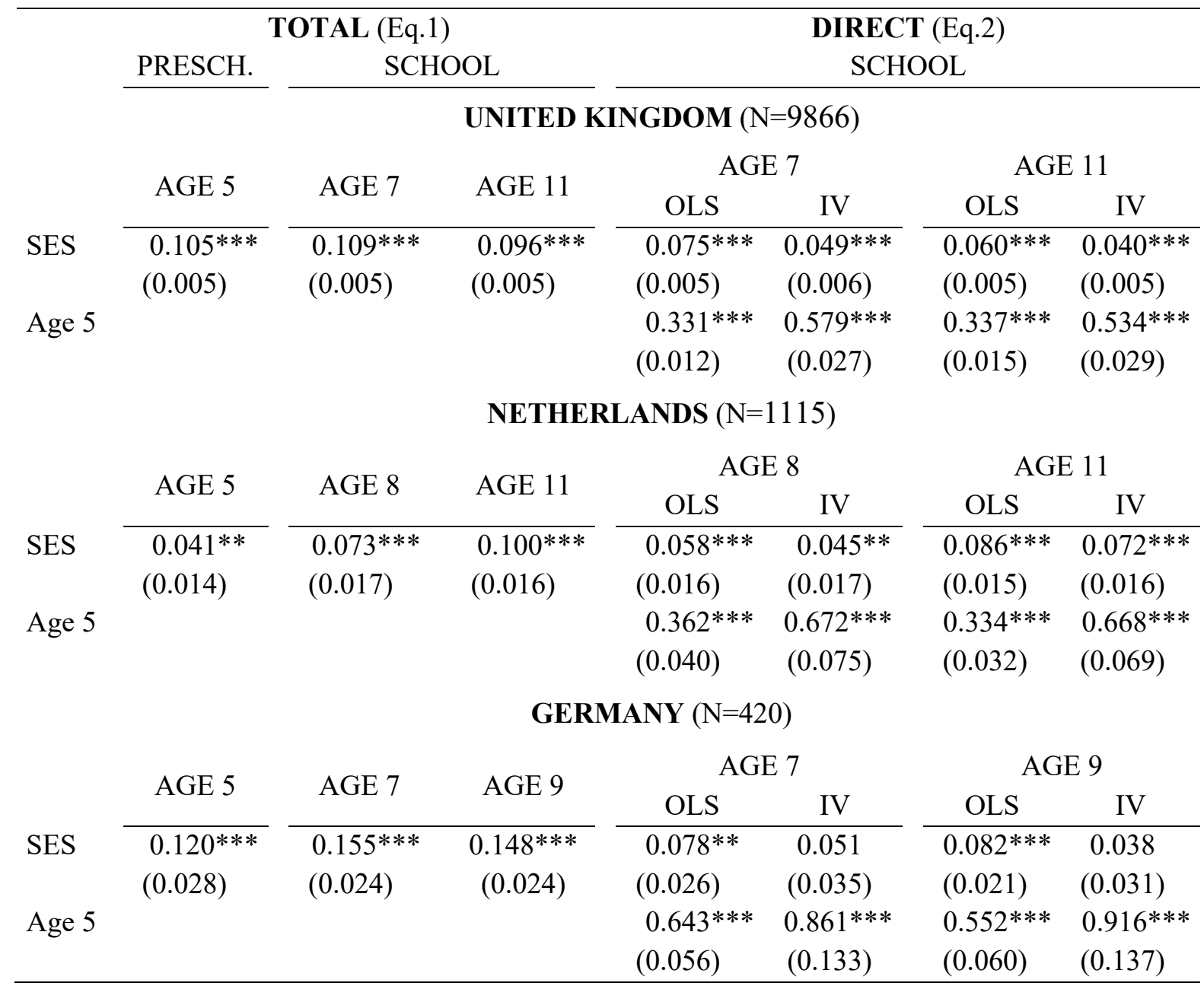

Notes: Dependent variables are standardized test scores at different ages (see Table 1). SES $=$ parents' highest years of education. TOTAL: total effect of SES on achievement. DIRECT: conditional SES effect after controlling for standardized achievement scores at age 5 (preschool age). All models control for migration background. IV models: results from second stage estimation shown. Full models for Equations 1 and 2 (IV only) are available in Appendix B (Table B1 and B3). Standard errors in parentheses. Significance: $* \mathrm{p}<0.05, * * \mathrm{p}<0.01, * * * \mathrm{p}<0.001$. 
Table 3 Total and direct effects of SES on achievement by age using SES groups.

\begin{tabular}{|c|c|c|c|c|c|c|c|}
\hline & \multicolumn{3}{|c|}{ TOTAL (Eq.1) } & \multicolumn{4}{|c|}{ DIRECT (Eq.2) } \\
\hline & PRESCH. & \multicolumn{2}{|c|}{ SCHOOL } & \multicolumn{4}{|c|}{ SCHOOL } \\
\hline & \multirow{3}{*}{ AGE 5} & \multicolumn{4}{|c|}{ UNITED KINGDOM (N=9866) } & & \\
\hline & & \multirow{2}{*}{ AGE 7} & \multirow{2}{*}{ AGE 11} & \multicolumn{2}{|c|}{ AGE 7} & \multicolumn{2}{|c|}{ AGE 11} \\
\hline SES & & & & OLS & IV & OLS & IV \\
\hline Med. & $\begin{array}{l}0.289 * * * \\
(0.027)\end{array}$ & $\begin{array}{l}0.292^{* * * *} \\
(0.028)\end{array}$ & $\begin{array}{l}0.246^{* * * *} \\
(0.031)\end{array}$ & $\begin{array}{l}0.196^{* * * *} \\
(0.026)\end{array}$ & $\begin{array}{l}0.125^{* * *} \\
(0.029)\end{array}$ & $\begin{array}{l}0.148 * * * \\
(0.028)\end{array}$ & $\begin{array}{l}0.092 * * \\
(0.029)\end{array}$ \\
\hline High & $\begin{array}{l}0.594 * * * \\
(0.030)\end{array}$ & $\begin{array}{l}0.636^{* * *} \\
(0.030)\end{array}$ & $\begin{array}{l}0.551^{* * *} \\
0.029\end{array}$ & $\begin{array}{l}0.438^{* * *} \\
(0.030)\end{array}$ & $\begin{array}{l}0.292 * * * \\
(0.033)\end{array}$ & $\begin{array}{l}0.349 * * * \\
(0.027)\end{array}$ & $\begin{array}{l}0.232 * * * \\
(0.030)\end{array}$ \\
\hline \multirow[t]{3}{*}{ Age 5} & & & & $\begin{array}{l}0.333 \text { *** } \\
(0.012)\end{array}$ & $\begin{array}{l}0.580 * * * \\
(0.026)\end{array}$ & $\begin{array}{l}0.339 * * * \\
(0.015)\end{array}$ & $\begin{array}{l}0.536^{* * * *} \\
(0.029)\end{array}$ \\
\hline & \multicolumn{7}{|c|}{ NETHERLANDS (N=1115) } \\
\hline & $\triangle C E=5$ & 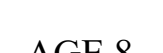 & $1 C D_{1} 11$ & $\mathrm{AG}$ & & $\mathrm{AG}$ & 11 \\
\hline \multirow{2}{*}{$\begin{array}{l}\text { SES } \\
\text { Med. }\end{array}$} & AUE J & AULO & AUE II & OLS & IV & OLS & IV \\
\hline & $\begin{array}{c}0.254^{* *} \\
(0.085)\end{array}$ & $\begin{array}{c}0.210^{*} \\
(0.100)\end{array}$ & $\begin{array}{l}0.285^{* * *} \\
(0.079)\end{array}$ & $\begin{array}{c}0.118 \\
(0.096)\end{array}$ & $\begin{array}{c}0.039 \\
(0.101)\end{array}$ & $\begin{array}{l}0.200^{* *} \\
(0.068)\end{array}$ & $\begin{array}{c}0.107 \\
(0.079)\end{array}$ \\
\hline High & $\begin{array}{l}0.345^{* * * *} \\
(0.092)\end{array}$ & $\begin{array}{l}0.491 * * * \\
(0.120)\end{array}$ & $\begin{array}{l}0.682 * * * \\
(0.111)\end{array}$ & $\begin{array}{l}0.367 * * \\
(0.113)\end{array}$ & $\begin{array}{c}0.260^{*} \\
(0.116)\end{array}$ & $\begin{array}{l}0.568 * * * \\
(0.101)\end{array}$ & $\begin{array}{l}0.455^{* * *} \\
(0.105)\end{array}$ \\
\hline \multirow[t]{3}{*}{ Age 5} & & & & $\begin{array}{l}0.362 * * * \\
(0.040)\end{array}$ & $\begin{array}{l}0.672 * * * \\
(0.075)\end{array}$ & $\begin{array}{l}0.332 * * * \\
(0.031)\end{array}$ & $\begin{array}{l}0.665 * * * \\
(0.072)\end{array}$ \\
\hline & \multicolumn{7}{|c|}{ GERMANY (N=420) } \\
\hline & AGE 5 & AGE 7 & AGE 9 & AG & & & E 9 \\
\hline \multirow{2}{*}{$\begin{array}{l}\text { SES } \\
\text { Med. }\end{array}$} & & & & OLS & IV & OLS & IV \\
\hline & $\begin{array}{l}0.660^{* *} \\
(0.197)\end{array}$ & $\begin{array}{l}0.841 * * * \\
(0.169)\end{array}$ & $\begin{array}{l}0.722 * * * \\
(0.177)\end{array}$ & $\begin{array}{l}0.429^{* *} \\
(0.142)\end{array}$ & $\begin{array}{c}0.282 \\
(0.147)\end{array}$ & $\begin{array}{l}0.370^{*} \\
(0.175)\end{array}$ & $\begin{array}{c}0.126 \\
(0.198)\end{array}$ \\
\hline High & $\begin{array}{l}0.935^{* * *} \\
(0.215)\end{array}$ & $\begin{array}{l}1.165^{* * *} \\
(0.184)\end{array}$ & $\begin{array}{l}1.122 * * * \\
(0.173)\end{array}$ & $\begin{array}{l}0.581 * * * \\
(0.160)\end{array}$ & $\begin{array}{c}0.372 \\
(0.205)\end{array}$ & $\begin{array}{l}0.624 * * * \\
(0.164)\end{array}$ & $\begin{array}{c}0.277 \\
(0.208)\end{array}$ \\
\hline Age 5 & & & & $\begin{array}{l}0.624 * * * \\
(0.061)\end{array}$ & $\begin{array}{l}0.847 * * * \\
(0.133)\end{array}$ & $\begin{array}{l}0.533 * * * \\
(0.063)\end{array}$ & $\begin{array}{l}0.903^{* * *} \\
(0.133)\end{array}$ \\
\hline
\end{tabular}

Notes: Dependent variables are standardized test scores at different ages (see Table 1). SES = parents' highest years of education grouped to low, medium, high; reference category is low SES. TOTAL: total effect of SES on achievement. DIRECT: conditional SES effect after controlling for standardized achievement scores at age 5 (preschool age). All models control for migration background. IV models: results from second stage estimation shown. Full models for Equations 1 and 2 (IV only) are available in Appendix B (Table B1 and B3). Standard errors in parentheses. Significance: ${ }^{*} \mathrm{p}<0.05,{ }^{* *} \mathrm{p}<0.01$, $* * * \mathrm{p}<0.001$. 
Table 4 Proportion (\%) of SES inequality in school-age achievement explained by SES inequality in preschool age (indirect channel).

\begin{tabular}{|c|c|c|c|c|}
\hline \multirow[b]{4}{*}{ SES measures } & \multicolumn{4}{|c|}{ \%INDIRECT } \\
\hline & \multicolumn{4}{|c|}{ UNITED KINGDOM } \\
\hline & \multicolumn{2}{|c|}{ AGE 7} & \multicolumn{2}{|c|}{ AGE 11} \\
\hline & OLS & IV & OLS & IV \\
\hline Metric & 31.7 & 55.5 & 36.9 & 58.5 \\
\hline \multicolumn{5}{|l|}{ Groups } \\
\hline Med. vs low & 32.9 & 57.2 & 39.8 & 62.8 \\
\hline \multirow[t]{4}{*}{ High vs low } & 31.1 & 54.2 & 36.6 & 57.8 \\
\hline & \multicolumn{4}{|c|}{ NETHERLANDS } \\
\hline & \multicolumn{2}{|c|}{ AGE 8} & \multicolumn{2}{|c|}{ AGE 11} \\
\hline & OLS & IV & OLS & IV \\
\hline Metric & 20.5 & 38.4 & 14.0 & 28.0 \\
\hline \multicolumn{5}{|l|}{ Groups } \\
\hline Med. vs low & 43.8 & 81.4 & 29.8 & 62.4 \\
\hline \multirow[t]{4}{*}{ High vs low } & 25.2 & 47.0 & 16.7 & 33.3 \\
\hline & \multicolumn{4}{|c|}{ GERMANY } \\
\hline & \multicolumn{2}{|c|}{ AGE 7} & \multicolumn{2}{|c|}{ AGE 9} \\
\hline & OLS & IV & OLS & IV \\
\hline Metric & 50.0 & 66.9 & 44.9 & 74.5 \\
\hline \multicolumn{5}{|l|}{ Groups } \\
\hline Med. vs low & 49.0 & 66.5 & 48.7 & 82.6 \\
\hline High vs low & 50.1 & 68.0 & 44.4 & 75.3 \\
\hline
\end{tabular}

Notes: \% explained by the direct channel is 100-indirect. SES measured as a metric and a group variable. OLS = naïve estimation. IV = accounting for measurement error through instrumental variable estimation. 
Figures

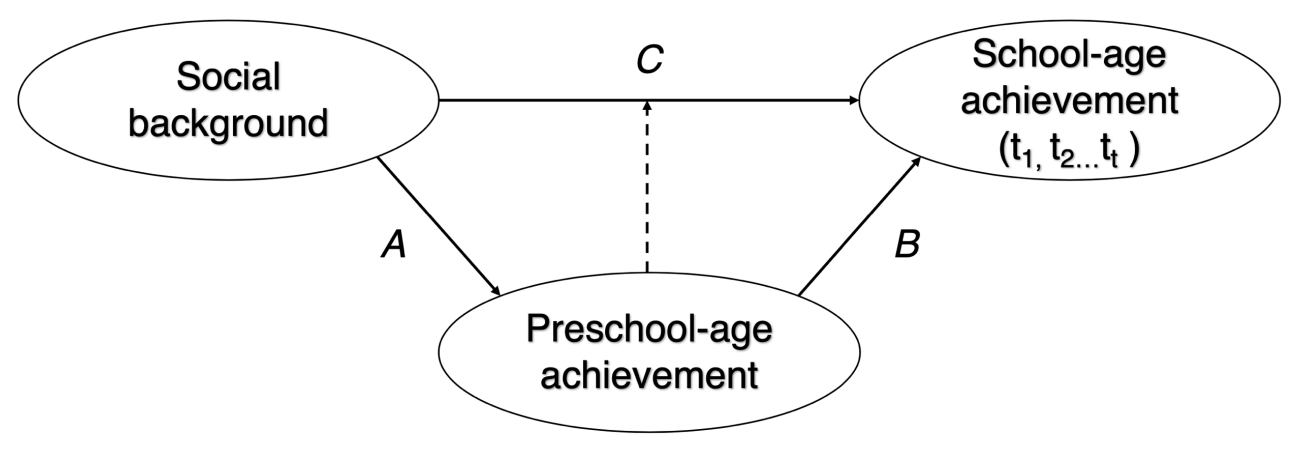

Figure 1. Direct and indirect paths to SES inequality in school-age achievement. 


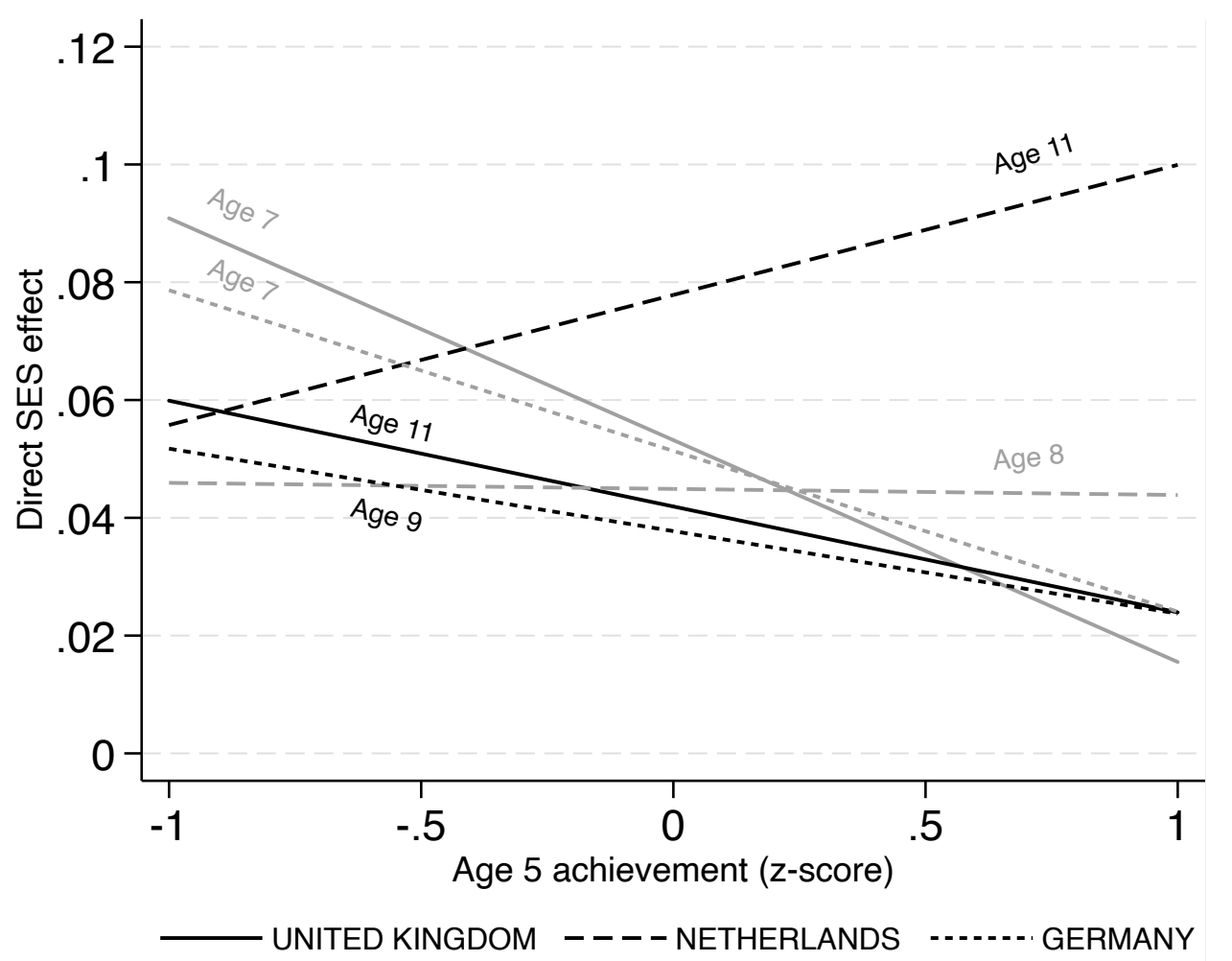

Figure 2. IV regression models (Equation 3): Direct SES-achievement association over primary schooling along the distribution of preschool-age achievement, separately by country.

Notes: interaction terms between preschool-age achievement and SES are statistically significant only in the UK (see Table B5). 


\section{Appendix A. Technical Details}

\section{A1. Weighting strategy}

Our weighting approach adjusts for survey design and selective panel attrition. We give a nontechnical description here, but the details (and the syntax) regarding all procedures involved are available upon request.

The respective surveys provide design weights that adjust the marginal distribution of samples' characteristics on the first measurement occasion to the target populations of interest. Starting from the initial representative unbalanced samples, we computed the inverse of the probability of being included in the following measurement point. The inversed probabilities are predicted from logistic models that regress participation on sex, migration background, and parental education interacted with the standardized scores in the first measurement occasion. The inverse of the same conditional probabilities is then computed between the second and the last measurement occasion. The procedure adjusts for selective attrition by assigning higher weights to children with higher probabilities of dropping out of the samples (for example, children from low SES and poor performances). In the MCS, the logit models used to predict the probability of participation include also ancillary variables to mimic the official MCS weighting strategy. For the same reason, in the MCS only, missing data on cognitive assessment were imputed in each wave via multiple imputation (we used five imputations; logit models were estimated for all imputed datasets and average predicted probabilities were used for weighting).

In all countries, we use response propensity score stratification to avoid large adjustment factors due to very low conditional probabilities. This procedure is quite robust to misspecification in the weighting model since it uses the actual response rate of the groups defined by the percentiles of the conditional probabilities rather than the conditional probabilities themselves (Chen, Gelman, Tracy, Norris, \& Galea, 2012). Survey design weights and the inversed conditional probabilities are eventually combined (multiplied) and applied to the balanced samples throughout the analyses. 


\section{A2. Construction of standardized scores ('residualization')}

A general issue in the construction of standardized scores is that children vary slightly in their age although tested in the same wave. Systematic age-variations by SES may confound the measurement of social inequality in achievement because age influences itself the process of cognitive development. Hence, we rule out possible age-variations at the test day before the standardization via residualization.

In general, the procedure involves regressing the raw test score $\left(r_{i t}\right)$ of the child $i$ on a cubic function of age at the test day separately in each wave $t$ :

$$
\begin{gathered}
r_{i t}=\alpha_{t}+b_{1} A g e_{i t}+b_{2} A g e_{i t}^{2}+b_{3} A g e_{i t}^{3}+e_{i t} \\
\text { for } t \in\{1,2,3\}
\end{gathered}
$$

After estimating equation A2.1, we, first, compute predicted scores $\hat{r}_{i t}$ and, second, the residuals $\hat{\delta}_{i t}=r_{i t}-\hat{r}_{i t}$. Residuals $\hat{\delta}_{i t}$ are standardized in a third step to construct the z-score variables:

$$
\begin{aligned}
& z_{i t}=\frac{\hat{\delta}_{i t}-\operatorname{mean}\left(\hat{\delta}_{t}\right)}{\operatorname{sd}\left(\hat{\delta}_{t}\right)} \\
& \text { for } t \in\{1,2,3\}
\end{aligned}
$$

Note that by construction those standardized residuals resemble relative differences in the language proficiency among equally-aged children - i.e. children at the same developmental stage - in each wave and country (see Bradbury et al. 2015 or Skopek and Passaretta 2018 for similar applications). We used weights throughout the construction of standardized scores. 


\section{A3. IV estimation: first- and second-stage equations}

In the NEPS and COOL, we instrumented z-standardized language scores at age 5 using zstandardized test scores for scientific literacy (NEPS) and arithmetic (COOL) measured in the same wave (age 5) but on a different day. Following Bradbury and colleagues (2015), in the MCS, we instrumented z-standardized language scores at age 5 using z-standardized test scores for vocabulary measured in the previous wave (age 3). We implemented the IV via two-stage least squares estimation using ivregress 2 sls in Stata. We use weights to ensure representativity at the national level. Equation 1 and 2 are estimated both using SES a metric and a group variable (notation refers to the SES as metric variable).

\section{Equation 2}

The first stage equation uses instrument variable $I$ to predict the z-score of the child $i$ at time 1. It is formulated as follows:

$$
z_{i 1}=\lambda_{0}+\lambda_{1} S E S_{i}+\lambda_{2} M I G_{i}+\lambda_{3} I_{i}+v_{i 1}
$$

Predicted z-scores $\hat{z}_{i 1}$ are plugged into the structural equation in the second stage:

$$
\begin{gathered}
z_{i t}=\beta_{0 t}+\beta_{1 t} S E S_{i}+\beta_{2 t} M I G_{i}+\beta_{3 t} \hat{z}_{i 1}+\epsilon_{i t} \\
\text { for } t \in\{2,3\}
\end{gathered}
$$

\section{Equation 3}

Equation 3 defines an interaction. Therefore, first stage equations are defined as

$$
\begin{gathered}
z_{i 1}=\lambda_{0}+\lambda_{1} S E S_{i}+\lambda_{2} M I G_{i}+\lambda_{3} I_{i}+\lambda_{4}\left(I_{i} \times S E S_{i}\right)+v_{i 1} \\
\text { and } \\
z_{i 1} S E S_{i}=\lambda_{0}+\lambda_{1} S E S_{i}+\lambda_{2} M I G_{i}+\lambda_{3} I_{i}+\lambda_{4}\left(I_{i} \times S E S_{i}\right)+v_{i 1} .
\end{gathered}
$$

The corresponding second stage equation uses predicted values for $z_{i 1}$ and $z_{i 1} S E S$ :

$$
z_{i t}=\gamma_{0 t}+\gamma_{1 t} M I G_{i}+\gamma_{2 t} S E S_{i}+\gamma_{3 t} \hat{z}_{i 1}+\gamma_{4 t} \bar{z}_{\imath 1} \overline{S E} S_{l}+\epsilon_{i t}
$$


Appendix B. Tables

Table B1. Equation 1 (metric SES) - Full model and coefficients (Table 2, TOTAL).

\begin{tabular}{|c|c|c|c|}
\hline & PRESCHOOL & $\mathrm{SC}$ & \\
\hline & \multicolumn{3}{|c|}{ UNITED KINGDOM (N=9866) } \\
\hline & AGE 5 & AGE 7 & AGE 11 \\
\hline \multirow[t]{2}{*}{ SES } & $0.105 * * *$ & $0.109 * * *$ & $0.096 * * *$ \\
\hline & $(0.005)$ & $(0.005)$ & $(0.005)$ \\
\hline \multirow[t]{2}{*}{ Migration (yes) } & $-0.686 * * *$ & $0.110^{* *}$ & -0.005 \\
\hline & $(0.065)$ & $(0.035)$ & $(0.057)$ \\
\hline \multirow[t]{4}{*}{ Constant } & $-1.286 * * *$ & $-1.454 * * *$ & $-1.259 * * *$ \\
\hline & $(0.066)$ & $(0.070)$ & $(0.075)$ \\
\hline & \multicolumn{3}{|c|}{ NETHERLANDS $(\mathrm{N}=1115)$} \\
\hline & AGE 5 & AGE 8 & AGE 11 \\
\hline \multirow[t]{2}{*}{ SES } & $0.041 * *$ & $0.073 * * *$ & $0.100 * * *$ \\
\hline & $(0.014)$ & $(0.017)$ & $(0.016)$ \\
\hline \multirow[t]{2}{*}{ Migration (yes) } & $-0.620 * * *$ & $-0.438 * * *$ & $-0.329 * *$ \\
\hline & $(0.116)$ & $(0.126)$ & $(0.124)$ \\
\hline \multirow[t]{4}{*}{ Constant } & $-0.467 *$ & $-0.973 * * *$ & $-1.395 * * *$ \\
\hline & $(0.222)$ & $(0.267)$ & $(0.244)$ \\
\hline & \multicolumn{3}{|c|}{ GERMANY (N=420) } \\
\hline & AGE 5 & AGE 7 & AGE 9 \\
\hline \multirow[t]{2}{*}{ SES } & $0.120 * * *$ & $0.155^{* * *}$ & $0.148 * * *$ \\
\hline & $(0.028)$ & $(0.024)$ & $(0.024)$ \\
\hline \multirow[t]{2}{*}{ Migration (yes) } & $-0.742 * * *$ & $-0.454 * *$ & $-0.450^{*}$ \\
\hline & $(0.163)$ & $(0.163)$ & $(0.182)$ \\
\hline \multirow[t]{2}{*}{ Constant } & $-1.386 * *$ & $-1.937 * * *$ & $-1.844 * * *$ \\
\hline & $(0.429)$ & $(0.343)$ & $(0.346)$ \\
\hline
\end{tabular}

Notes: Dependent variables are standardized test scores at different ages (see Table 1). SES $=$ parents' highest years of education. Standard errors in parentheses. Significance: $* \mathrm{p}<0.05, * * \mathrm{p}<0.01, * * *$ $\mathrm{p}<0.001$. 
Table B2. Equation 1 (categorical SES) - Full model and coefficients (Table 3, TOTAL).

\begin{tabular}{|c|c|c|c|}
\hline \multirow[b]{3}{*}{ SES } & PRESCHOOL & \multicolumn{2}{|c|}{ SCHOOL } \\
\hline & \multicolumn{3}{|c|}{ UNITED KINGDOM (N=9866) } \\
\hline & AGE 5 & AGE 7 & AGE 11 \\
\hline \multirow[t]{2}{*}{ Medium } & $0.289 * * *$ & $0.292 * * *$ & $0.246^{* * *}$ \\
\hline & $(0.027)$ & $(0.028)$ & $(0.031)$ \\
\hline \multirow[t]{2}{*}{ High } & $0.594 * * *$ & $0.636 * * *$ & $0.551 * * *$ \\
\hline & $(0.031)$ & $(0.030)$ & $(0.029)$ \\
\hline \multirow[t]{2}{*}{ Migration (yes) } & $-0.676 * * *$ & $0.118 * * *$ & 0.00241 \\
\hline & $(0.066)$ & $(0.035)$ & $(0.057)$ \\
\hline \multirow[t]{3}{*}{ Constant } & $-0.131 * * *$ & $-0.251 * * *$ & $-0.202 * * *$ \\
\hline & $(0.019)$ & $(0.023)$ & $(0.028)$ \\
\hline & \multicolumn{3}{|c|}{ NETHERLANDS (N=1115) } \\
\hline SES & AGE 5 & AGE 8 & AGE 11 \\
\hline \multirow[t]{2}{*}{ Medium } & $0.254 * *$ & $0.210 *$ & $0.285 * * *$ \\
\hline & $(0.085)$ & $(0.100)$ & $(0.079)$ \\
\hline \multirow[t]{2}{*}{ High } & $0.345^{* * *}$ & $0.491 * * *$ & $0.682 * * *$ \\
\hline & $(0.092)$ & $(0.120)$ & $(0.111)$ \\
\hline \multirow[t]{2}{*}{ Migration (yes) } & $-0.613 * * *$ & $-0.478 * * *$ & $-0.384 * *$ \\
\hline & $(0.114)$ & $(0.127)$ & $(0.119)$ \\
\hline \multirow[t]{3}{*}{ Constant } & -0.097 & -0.164 & $-0.286 * * *$ \\
\hline & $(0.089)$ & $(0.095)$ & $(0.078)$ \\
\hline & \multicolumn{3}{|c|}{ GERMANY (N=420) } \\
\hline SES & AGE 5 & AGE 7 & AGE 9 \\
\hline \multirow[t]{2}{*}{ Medium } & $0.660 * *$ & $0.841 * * *$ & $0.722 * * *$ \\
\hline & $(0.197)$ & $(0.169)$ & $(0.177)$ \\
\hline \multirow[t]{2}{*}{ High } & $0.935 * * *$ & $1.165^{* * *}$ & $1.122 * * *$ \\
\hline & $(0.215)$ & $(0.184)$ & $(0.173)$ \\
\hline \multirow[t]{2}{*}{ Migration (yes) } & $-0.662 * * *$ & $-0.366^{*}$ & $-0.379 *$ \\
\hline & $(0.161)$ & $(0.155)$ & $(0.163)$ \\
\hline \multirow[t]{2}{*}{ Constant } & -0.358 & $-0.592 * * *$ & $-0.525 * * *$ \\
\hline & $(0.210)$ & $(0.159)$ & $(0.154)$ \\
\hline
\end{tabular}

Notes: Dependent variables are standardized test scores at different ages (see Table 1). SES = parents' highest years of education grouped to low, medium, high; reference category is low SES. Standard errors in parentheses. Significance: $* \mathrm{p}<0.05, * * \mathrm{p}<0.01, * * * \mathrm{p}<0.001$. 
Table B3. Equation 2 (metric SES) - IV first and second stage, full models (Table 2, DIRECT).

\begin{tabular}{|c|c|c|c|c|c|c|}
\hline \multicolumn{7}{|c|}{ FIRST STAGES $\left(z_{l}\right)$} \\
\hline & \multicolumn{2}{|c|}{ UNITED KINGDOM } & \multicolumn{2}{|c|}{ NETHERLANDS } & \multicolumn{2}{|c|}{ GERMANY } \\
\hline \multirow[t]{2}{*}{ SES } & \multicolumn{2}{|c|}{$0.061^{* * *}$} & \multicolumn{2}{|c|}{0.018} & \multicolumn{2}{|c|}{$0.059^{* *}$} \\
\hline & \multicolumn{2}{|c|}{$(0.004)$} & \multicolumn{2}{|c|}{$(0.012)$} & \multicolumn{2}{|c|}{$(0.024)$} \\
\hline \multicolumn{7}{|l|}{ Migration } \\
\hline \multirow[t]{2}{*}{ (yes) } & \multicolumn{2}{|c|}{$-0.290 * * *$} & \multicolumn{2}{|c|}{$-0.433 * * *$} & \multicolumn{2}{|c|}{$-0.498 * *$} \\
\hline & & \multicolumn{2}{|c|}{$(0.097)$} & \multicolumn{2}{|c|}{$(0.154)$} \\
\hline \multirow[t]{2}{*}{ Instrument } & \multicolumn{2}{|c|}{$0.505 * * *$} & \multicolumn{2}{|c|}{$0.479 * * *$} & \multicolumn{2}{|c|}{$0.464 * * *$} \\
\hline & \multicolumn{2}{|c|}{$(0.013)$} & \multicolumn{2}{|c|}{$(0.028)$} & \multicolumn{2}{|c|}{$(0.074)$} \\
\hline \multirow[t]{2}{*}{ Constant } & \multirow{2}{*}{\multicolumn{2}{|c|}{$\begin{array}{l}-0.769 * * * \\
(0.058)\end{array}$}} & \multirow{2}{*}{\multicolumn{2}{|c|}{$\begin{array}{l}-0.165 \\
(0.187)\end{array}$}} & \multirow{2}{*}{\multicolumn{2}{|c|}{$\begin{array}{l}-0.644 \\
(0.358)\end{array}$}} \\
\hline & & & & & & \\
\hline F-statistic & \multicolumn{2}{|l|}{646} & \multicolumn{2}{|l|}{294} & \multicolumn{2}{|l|}{31} \\
\hline \multicolumn{7}{|c|}{ SECOND STAGES $\left(z_{t}\right)$} \\
\hline & AGE 7 & AGE 11 & AGE 8 & AGE 11 & AGE 7 & AGE 9 \\
\hline SES & $0.049 * * *$ & $0.040 * * *$ & $0.045^{* *}$ & $0.072 * * *$ & 0.051 & 0.038 \\
\hline & $(0.006)$ & $(0.005)$ & $(0.017)$ & $(0.016)$ & $(0.035)$ & $(0.031)$ \\
\hline Migration & & & & & & \\
\hline (yes) & $0.508 * * *$ & $0.361 * * *$ & -0.021 & 0.085 & 0.185 & 0.230 \\
\hline & $(0.040)$ & $(0.042)$ & $(0.122)$ & $(0.117)$ & $(0.160)$ & $(0.209)$ \\
\hline Age $5\left(z_{1}\right)$ & $0.579 * * *$ & $0.534 * * *$ & $0.672 * * *$ & $0.668 * * *$ & $0.861 * * *$ & $0.916 * * *$ \\
\hline & $(0.027)$ & $(0.029)$ & $(0.075)$ & $(0.069)$ & $(0.133)$ & $(0.137)$ \\
\hline Constant & $-0.710 * * *$ & $-0.573 * * *$ & $-0.659 * *$ & $-1.083 * * *$ & -0.743 & -0.575 \\
\hline & $(0.074)$ & $(0.073)$ & $(0.254)$ & $(0.238)$ & $(0.455)$ & $(0.439)$ \\
\hline
\end{tabular}

Notes: The reported F-statistic is for a test of excluded instruments (instrument relevance test). SES $=$ parents' highest years of education. Standard errors in parentheses. Significance: ${ }^{*} p<0.05,{ }^{* *} p<0.01$, $* * * \mathrm{p}<0.001$. 
Table B4. Equation 2 (categorical SES) - IV first and second stage, full models (Table 3, DIRECT).

\begin{tabular}{|c|c|c|c|c|c|c|}
\hline \multicolumn{7}{|c|}{ FIRST STAGES ( $Y=$ Age 5 zscore $)$} \\
\hline & \multicolumn{2}{|c|}{ UNITED KINGDOM } & \multicolumn{2}{|c|}{ NETHERLANDS } & \multicolumn{2}{|c|}{ GERMANY } \\
\hline \multicolumn{7}{|l|}{ SES } \\
\hline Medium & \multicolumn{2}{|c|}{$0.164 * * *$} & \multicolumn{2}{|c|}{$0.181^{*}$} & \multicolumn{2}{|c|}{$0.410^{*}$} \\
\hline & \multicolumn{2}{|c|}{$(0.025)$} & \multicolumn{2}{|c|}{$(0.074)$} & \multicolumn{2}{|c|}{$(0.160)$} \\
\hline \multirow[t]{2}{*}{ High } & \multicolumn{2}{|c|}{$0.346^{* * *}$} & \multicolumn{2}{|c|}{$0.174 *$} & \multicolumn{2}{|c|}{$0.493 * *$} \\
\hline & \multicolumn{2}{|c|}{$(0.027)$} & \multicolumn{2}{|c|}{$(0.079)$} & \multicolumn{2}{|c|}{$(0.175)$} \\
\hline \multirow[t]{2}{*}{ Migration (yes) } & \multicolumn{2}{|c|}{$-0.282 * * *$} & \multicolumn{2}{|c|}{$-0.417 * * *$} & \multicolumn{2}{|c|}{$-0.445 * *$} \\
\hline & \multicolumn{2}{|c|}{$(0.039)$} & \multicolumn{2}{|c|}{$(0.094)$} & \multicolumn{2}{|c|}{$(0.149)$} \\
\hline \multirow[t]{2}{*}{ Instrument } & \multicolumn{2}{|c|}{$0.510 * * *$} & \multicolumn{2}{|c|}{$0.477 * * *$} & \multicolumn{2}{|c|}{$0.449 * * *$} \\
\hline & \multicolumn{2}{|c|}{$(0.013)$} & \multicolumn{2}{|c|}{$(0.028)$} & \multicolumn{2}{|c|}{$(0.072)$} \\
\hline Constant & -0.09 & & -0.0 & & -0.1 & \\
\hline & $(0.01$ & & $(0.0$ & & $(0.1$ & \\
\hline F-statistic & 477 & & 285 & & 29 & \\
\hline & & & ND STAGE & & & \\
\hline & AGE 7 & AGE 11 & AGE 8 & AGE 11 & AGE 7 & AGE 9 \\
\hline SES & & & & & & \\
\hline Medium & $0.125 * * *$ & $0.092 * *$ & 0.039 & 0.107 & 0.282 & 0.126 \\
\hline & $(0.029)$ & $(0.029)$ & $(0.101)$ & $(0.079)$ & $(0.147)$ & $(0.198)$ \\
\hline High & $0.292 * * *$ & $0.232 * * *$ & $0.260^{*}$ & $0.455^{* * *}$ & 0.372 & 0.277 \\
\hline & $(0.033)$ & $(0.030)$ & $(0.116)$ & $(0.105)$ & $(0.205)$ & $(0.208)$ \\
\hline Migration (yes) & $0.510 * * *$ & $0.365^{* * *}$ & -0.066 & 0.033 & 0.195 & 0.219 \\
\hline & $(0.040)$ & $(0.042)$ & $(0.124)$ & $(0.115)$ & $(0.171)$ & $(0.218)$ \\
\hline Age $5\left(z_{1}\right)$ & $0.580 * * *$ & $0.536 * * *$ & $0.672 * * *$ & $0.665^{* * *}$ & $0.847 * * *$ & $0.903 * * *$ \\
\hline & $(0.026)$ & $(0.029)$ & $(0.075)$ & $(0.072)$ & $(0.133)$ & $(0.133)$ \\
\hline Constant & $-0.175 * * *$ & $-0.132 * * *$ & -0.099 & $-0.217 * *$ & $-0.290 *$ & -0.202 \\
\hline & $(0.022)$ & $(0.026)$ & $(0.092)$ & $(0.076)$ & $(0.119)$ & $(0.190)$ \\
\hline
\end{tabular}

Notes: The reported F-statistic is for a test of excluded instruments (instrument relevance test). SES = parents' highest years of education grouped to low, medium, high; reference category is low SES. Standard errors in parentheses. Significance: ${ }^{*} \mathrm{p}<0.05,{ }^{* *} \mathrm{p}<0.01, * * * \mathrm{p}<0.001$. 
Table B5. Equation 3 - IV first and second stage, full models (Figure 2).

\begin{tabular}{|c|c|c|c|c|c|c|}
\hline \multicolumn{7}{|c|}{ FIRST STAGES } \\
\hline & \multicolumn{2}{|c|}{ UNITED KINGDOM } & \multicolumn{2}{|c|}{ NETHERLANDS } & \multicolumn{2}{|c|}{ GERMANY } \\
\hline & $z_{1}$ & $z_{l} S E S$ & $z_{1}$ & $z_{l} S E S$ & $z_{1}$ & $z_{1} S E S$ \\
\hline \multirow[t]{2}{*}{ SES } & $0.061 * * *$ & $0.876^{* * *}$ & 0.017 & 0.121 & $0.059^{*}$ & $0.792^{*}$ \\
\hline & $(0.005)$ & $(0.073)$ & $(0.012)$ & $(0.150)$ & $(0.025)$ & $(0.318)$ \\
\hline \multirow[t]{2}{*}{ Migration (yes) } & $-0.289 * * *$ & $-4.076^{* * *}$ & $-0.432 * * *$ & $-5.862 * * *$ & $-0.417 *$ & $-4.937 *$ \\
\hline & $(0.039)^{* * *}$ & $(0.514)$ & $(0.098)$ & $(1.369)$ & $(0.177)$ & $(2.244)$ \\
\hline \multirow[t]{2}{*}{ Instrument } & $0.454 * * *$ & -1.440 & $0.507 * *$ & -1.306 & $1.023^{*}$ & 5.199 \\
\hline & $(0.055)$ & $(0.787)$ & $(0.168)$ & $(1.977)$ & $(0.412)$ & $(4.943)$ \\
\hline \multirow[t]{2}{*}{ Instrument $x$ SES } & 0.004 & $0.615 * * *$ & -0.002 & $0.567 * * *$ & -0.040 & .071 \\
\hline & $(0.004)$ & $(0.064)$ & $(0.011)$ & $(0.140)$ & $(0.026)$ & $(0.320)$ \\
\hline \multirow[t]{2}{*}{ Constant } & $-0.767 * * *$ & $-10.682 * * *$ & -0.159 & -0.308 & -0.621 & -7.943 \\
\hline & $(0.059)$ & $(0.858)$ & $(0.183)$ & $(2.144)$ & $(0.357)$ & (4.494) \\
\hline \multirow[t]{3}{*}{ F-statistic } & 487 & 510 & 148 & 147 & 30 & 37 \\
\hline & \multicolumn{4}{|c|}{ SECOND STAGES } & & \\
\hline & AGE 7 & AGE 11 & AGE 8 & AGE 11 & AGE 7 & AGE 9 \\
\hline \multirow[t]{2}{*}{ SES } & $0.053 * * *$ & $0.042 * * *$ & $0.045 * *$ & $0.078 * * *$ & 0.051 & 0.038 \\
\hline & $(0.006)$ & $(0.005)$ & $(0.017)$ & $(0.016)$ & $(0.036)$ & $(0.032)$ \\
\hline \multirow[t]{2}{*}{ Migration (yes) } & $0.489 * * *$ & $0.352 * * *$ & -0.020 & 0.064 & 0.234 & 0.256 \\
\hline & $(0.042)$ & $(0.041)$ & $(0.125)$ & $(0.121)$ & $(0.177)$ & $(0.237)$ \\
\hline \multirow[t]{2}{*}{ Age $5\left(z_{1}\right)$} & $1.074 * * *$ & $0.770 * * *$ & $0.688^{* *}$ & 0.341 & $1.225^{*}$ & 1.102 \\
\hline & $(0.108)$ & $(0.106)$ & $(0.242)$ & $(0.249)$ & $(.556)$ & $(0.527)$ \\
\hline \multirow[t]{2}{*}{ Age $5\left(\mathrm{z}_{1} \mathrm{SES}\right)$} & $-0.038 * * *$ & $-0.018 * *$ & -0.001 & 0.022 & -0.027 & -0.014 \\
\hline & $(0.007)$ & $(0.007)$ & $(0.017)$ & $(0.017)$ & $(-0.040)$ & $(0.036)$ \\
\hline \multirow[t]{2}{*}{ Constant } & $-0.745^{* * *}$ & $-0.590 * * *$ & $-0.655^{*}$ & $-1.168 * * *$ & -0.724 & -0.565 \\
\hline & $(0.075)$ & $(0.073)$ & $(0.256)$ & $(0.236)$ & $(0.459)$ & $(0.437)$ \\
\hline
\end{tabular}

Notes: The reported F-statistic is for a test of excluded instruments (instrument relevance test). SES = parents' highest years of education. Standard errors in parentheses. Significance: ${ }^{*} \mathrm{p}<0.05, * * \mathrm{p}<0.01, * * * \mathrm{p}<0.001$. 